\title{
Extended Variational Formulation for Heterogeneous Partial Differential Equations
}

\author{
Pablo Blanco • Paola Gervasio • Alfio Quarteroni
}

\begin{abstract}
We address the coupling of an advection equation with a diffusionadvection equation, for solutions featuring boundary layers. We consider nonoverlapping domain decompositions and we face up the heterogeneous problem using an extended variational formulation. We prove the equivalence between the latter formulation and a treatment based on a singular perturbation theory. An exhaustive comparison in terms of solution and computational efficiency between these formulations is carried out.
\end{abstract}

2010 Mathematical subject classification: 35Q35; 65N55; 65N35; 65F08.

Keywords: coupled elliptic-hyperbolic; advection-diffusion-reaction; domain decomposition; spectral elements; preconditioning.

\section{Introduction}

Subdomain splitting is an interesting path towards multiphysics, i.e., the use of mathematical models based on different kinds of partial differential equations to address physical problems of heterogeneous nature in different subregions of a given computational domain. In this presentation we focus on the framework of advection-diffusion equations with boundary layer solutions. The complete elliptic advection-diffusion problem is solved only in a small subdomain embodying the layer, while the reduced hyperbolic model, that is obtained by neglecting the diffusion term, is used on the remainder of the computational domain.

Gastaldi et al. (see $[18,17]$ ) analyzed this problem and they derived a suitable set of matching conditions at the interface between subdomains, which guarantee the well posedness of the heterogeneous problem. Such conditions express the continuity of the velocity field across the inflow part of the interface (i.e., the part of interface which is an inflow for the hyperbolic domain) and the continuity of the fluxes (i.e., the conormal derivatives associated to the differential operators) across the whole interface. If properly split, these

\footnotetext{
Pablo Blanco

LNCC, Laboratório Nacional de Computação Científica and INCT-MACC, Instituto Nacional de Ciência e Tecnologia em Medicina Assistida por Computação Científica, Av. Getúlio Vargas 333, Quitandinha, 25651075 Petrópolis, RJ, Brazil

E-mail: pjblanco@lncc.br.

Paola Gervasio

Department of Mathematics, University of Brescia, via Valotti, 9. 25133 Brescia, Italy

E-mail: gervasio@ing.unibs.it.

Alfio Quarteroni

MOX, Department of Mathematics "F. Brioschi", Politecnico di Milano, via Bonardi, 9. 20133 Milano, Italy SB-MATHICSE-CMCS École Polytechnique Fédérale de Lausanne, CH-1015 Lausanne, Switzerland

E-mail: alfio.quarteroni@epfl.ch.
} 
conditions can be used to numerically solve the problem through a Dirichlet/Neumann-like algorithm. Different kinds of boundary conditions were derived in [11, 15].

The set of interface conditions proposed in [18] can be formulated in two ways which are equivalent at continuous level and yield two equivalent Steklov-Poincaré formulations, but they differ one another in imposing the continuity of fluxes on the interface. The first set of interface conditions (IC1) enforces the continuity of fluxes on the whole interface, while the second set (IC2) exploits the continuity of traces across the inflow interface, so that, there, the continuity of fluxes is achieved by imposing null normal derivative of the elliptic solution. A special attention is given in this work to the comparison of the two sets of interface conditions IC1 and IC2. At discrete level, the formulation of flux continuity on the inflow interface is responsible for the efficiency of the corresponding approach, the interface conditions IC2 perform better than IC1, mainly when the viscosity is small.

More recently $([3,4])$, an extended variational approach has been proposed to solve heterogeneous problems, including those coupled problems featuring different geometrical dimensions. The starting point of this approach is the reduction of the geometrical dimension of the problem in a part of the computational domain, motivated by the need of reducing the computational cost in applications of practical interest. Such geometrical reduction entails different kinematic assumptions within the different subregions of the domain, so that a heterogeneous problem arises. This problem is then re-formulated globally (i.e., on the whole computational domain), by resorting to a saddle-point approach in which the constraint expresses the continuity of the solution across either the interface or a subset of it. The associated Lagrange multipliers are the fluxes across the interface, more precisely, the conormal derivatives associated to the differential operators defined in the different subdomains. Consequently, the matching conditions at the interface are identified by writing the Euler-Lagrange system associated to the saddle-point problem.

In this paper, we re-formulate the heterogeneous advection/advection-diffusion problem in terms of the extended variational formulation. How to choose the functional spaces and the bilinear forms is suggested by the well-posedness of the saddle-point problem. The first goal of this paper consists in finding out which interface conditions, alternative to those proposed in [18], are admissible and lead to a well-posed extended variational formulation. In Section 3, we prove that the saddle-point problem whose constraint enforces the continuity of the solution only across the inflow (and not on the whole) interface is well-posed and the associated Euler-Lagrange equations provide the same interface conditions given in [18].

In Section 4, the extended variational problem is re-formulated as an interface problem in terms of Steklov-Poincaré operators. Four possible coupling strategies are analyzed depending on how the interface unknowns are chosen, as traces of order zero (Dirichlet's) or one (Neumann's). They are named DD, NN, DN and ND, where the first letter identifies the kind of trace $(\mathrm{D}=$ Dirichlet, $\mathrm{N}=$ Neumann) used for the hyperbolic solution, the second one that for the elliptic problem.

In Section 5.3, we propose optimal preconditioners for the finite dimensional counterpart of the extended interface problems. Such preconditioners are built as inexact factorizations of the primal matrices in which the Schur complement matrix is replaced by its optimal preconditioner. No preconditioners for extended problems have been developed so far and it has been proved tat the proposed preconditioners have good properties. In all cases, the condition number of the preconditioned matrices are bounded from above independently of the discretization parameters (grid space and polynomial degree).

The second aim of this work is to set up a systematic comparison between the hetero- 
geneous approach $([18])$ and XVF, from the computational point of view. We discretize the PDE's by conforming Spectral Elements and we solve the interface problems by the preconditioned Bi-CGStab method [24].

The non-conforming discretization of the hyperbolic-elliptic heterogeneous problem is a matter of current research ([5]). A paper that deals with a similar class of problems (anisotropic semidefinite diffusion problems with advection) is [20], where the authors approximate the solution by Discontinuous Galerkin methods.

We compare the Steklov-Poincaré approach with all the proposed extended variational forms for what concerns accuracy, boundedness of the condition number of the preconditioned matrix, and computational efficiency. In order to analyze the accuracy, we measure trace and flux jumps across interface between elliptic and hyperbolic solutions. The best accurate approaches are those based on the Steklov-Poincaré equation and the Dirichlet-Dirichlet version of the XVF, while both Dirichlet-Neumann and Neumann-Neumann forms are illposed for advection-dominated problems when interface conditions IC1 are considered. From the computational point of view, the most efficient approaches are those based on the SteklovPoincaré equation, as they entail the lowest number of elliptic and hyperbolic subproblems at each preconditioned Bi-CGStab iteration.

In conclusion, XVF is a valid alternative to the heterogeneous form proposed in [18] from the theoretical point of view. Moreover, it is interesting to see that XVF provides the same set of interface conditions derived in [18] and then the latter approach strengthens the validity of the former one. At discrete level, Extended Variational Formulation is not so efficient as the Steklov-Poincaré approach, as a matter of fact the computational complexity of the augmented linear system associated to XVF is larger than that of Steklov-Poincaré equation.

An outline of the paper is as follows. In Section 2, we introduce the problem setting and resume known theoretical results about the well-posedness of the heterogeneous advection/advection-diffusion problem. In Section 3, we present and analyze the XVF of the heterogeneous problem, while in Section 4 we write the interface problem (XIP) associated to XVF. Section 5 is devoted to the discretization of the XIP, the development and analysis of suitable preconditioners for XIP and the numerical results comparing Steklov-Poincaré formulation and XIP.

\section{Problem setting}

We consider an open bounded domain $\Omega \subset \mathbb{R}^{d}(d=2,3)$ with Lipschitz boundary $\partial \Omega$, split into two open subsets $\Omega_{1}$ and $\Omega_{2}$ such that

$$
\bar{\Omega}=\bar{\Omega}_{1} \cup \bar{\Omega}_{2}, \quad \Omega_{1} \cap \Omega_{2}=\emptyset .
$$

Then, we denote by

$$
\Gamma=\partial \Omega_{1} \cap \partial \Omega_{2}
$$

the interface between the subdomains (see Fig. 2.1) and we assume that $\Gamma$ is of class $C^{1,1}$; $\stackrel{\circ}{\Gamma}$ denotes the interior of $\Gamma$.

Given two scalar functions $f$ and $b_{0}$ defined in $\Omega$, a positive function $\nu$ defined in $\Omega_{2} \cup \stackrel{\circ}{\Gamma}$, 


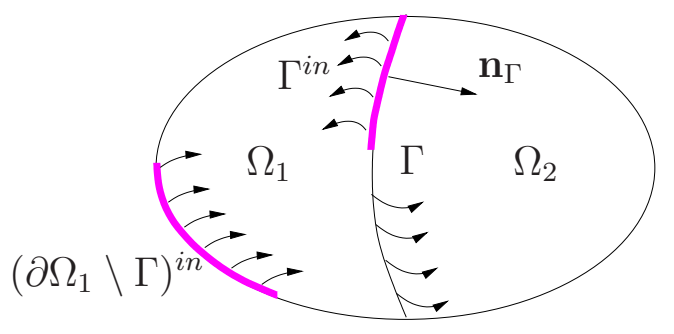

Figure 2.1. Example of a computational domain $\Omega \subset \mathbb{R}^{2}$ split into two disjoint subdomains

a $d$-dimensional vector valued function $\mathbf{b}$ defined in $\Omega$ satisfying the following inequalities:

$$
\begin{aligned}
& \exists \nu_{0} \in \mathbb{R}: \quad \nu(\mathbf{x}) \geqslant \nu_{0}>0, \quad \forall \mathbf{x} \in \Omega_{2} \cup \stackrel{\circ}{\Gamma} \\
& \exists \sigma_{0} \in \mathbb{R}: \quad b_{0}(\mathbf{x})+\frac{1}{2} \operatorname{divb}(\mathbf{x}) \geqslant \sigma_{0}>0, \quad \forall \mathbf{x} \in \Omega,
\end{aligned}
$$

we look for two functions $u_{1}$ and $u_{2}$ (defined in $\bar{\Omega}_{1}$ and $\bar{\Omega}_{2}$, respectively) such that $u_{1}$ satisfies the advection-reaction equation

$$
A_{1} u_{1} \equiv \operatorname{div}\left(\mathbf{b} u_{1}\right)+b_{0} u_{1}=f, \quad \text { in } \Omega_{1},
$$

while $u_{2}$ satisfies the advection-diffusion-reaction equation

$$
A_{2} u_{2} \equiv \operatorname{div}\left(-\nu \nabla u_{2}+\mathbf{b} u_{2}\right)+b_{0} u_{2}=f, \quad \text { in } \Omega_{2}
$$

For each subdomain, we distinguish between the external (or physical) boundary $\partial \Omega \cap$ $\partial \Omega_{k}=\partial \Omega_{k} \backslash \Gamma$ (for $\left.k=1,2\right)$ and the internal one (i.e. the interface) $\Gamma$. Let us denote by $\mathbf{n}_{k}$ the outward normal unit vector to $\partial \Omega_{k}$ and by $\mathbf{n}_{\Gamma}$ the normal unit vector to $\Gamma$ oriented from $\Omega_{1}$ to $\Omega_{2}$, so that $\mathbf{n}_{\Gamma}(\mathbf{x})=\mathbf{n}_{1}(\mathbf{x})=-\mathbf{n}_{2}(\mathbf{x}), \forall \mathbf{x} \in \Gamma$.

Moreover, for any non-empty subset $S \subseteq \partial \Omega_{1}$, we define

$$
\begin{gathered}
\text { the inflow part of } S: \quad S^{i n}=\left\{\mathbf{x} \in S: \mathbf{b}(\mathbf{x}) \cdot \mathbf{n}_{1}(\mathbf{x})<0\right\} \\
\text { the outflow part of } S: \quad S^{\text {out }}=\left\{\mathbf{x} \in S: \mathbf{b}(\mathbf{x}) \cdot \mathbf{n}_{1}(\mathbf{x}) \geqslant 0\right\} .
\end{gathered}
$$

Boundary conditions for problem (2.4) are assigned on the inflow boundary $\left(\partial \Omega_{1}\right)^{\text {in }}$. Then, we set homogeneous Dirichlet boundary conditions on the external boundaries

$$
u_{1}=0 \quad \text { on }\left(\partial \Omega_{1} \backslash \Gamma\right)^{i n}, \quad u_{2}=0 \quad \text { on } \partial \Omega_{2} \backslash \Gamma \text {. }
$$

A crucial issue is the setting of the interface (or transmission) conditions on $\Gamma$.

In [18], the heterogeneous problem (2.4), (2.5), (2.8) is closed with the following interface conditions (that are named IC1):

$$
u_{1}=u_{2} \quad \text { on } \Gamma^{\text {in }}, \quad-\mathbf{b} \cdot \mathbf{n}_{\Gamma} u_{1}=\nu \frac{\partial u_{2}}{\partial \mathbf{n}_{\Gamma}}-\mathbf{b} \cdot \mathbf{n}_{\Gamma} u_{2} \quad \text { on } \Gamma .
$$

They express the continuity of the velocity field across the inflow part of the interface and the continuity of the fluxes across the whole interface. 
The final formulation of the heterogeneous problem reads

$$
\begin{cases}\operatorname{div}\left(\mathbf{b} u_{1}\right)+b_{0} u_{1}=f & \text { in } \Omega_{1}, \\ \operatorname{div}\left(-\nu \nabla u_{2}+\mathbf{b} u_{2}\right)+b_{0} u_{2}=f & \text { in } \Omega_{2}, \\ u_{1}=0 & \text { on }\left(\partial \Omega_{1} \backslash \Gamma\right)^{i n} \\ u_{2}=0 & \text { on } \partial \Omega_{2} \backslash \Gamma \\ u_{1}=u_{2} & \text { on } \Gamma^{\text {in }} \\ \mathbf{b} \cdot \mathbf{n}_{\Gamma} u_{1}+\nu \frac{\partial u_{2}}{\partial \mathbf{n}_{\Gamma}}-\mathbf{b} \cdot \mathbf{n}_{\Gamma} u_{2}=0 & \text { on } \Gamma\end{cases}
$$

and its solution will be named heterogeneous solution (or else solution of the heterogeneous problem).

Note that the interface conditions (2.9) can be equivalently expressed as

$$
u_{1}=u_{2}, \quad \nu \frac{\partial u_{2}}{\partial \mathbf{n}_{\Gamma}}=0 \quad \text { on } \Gamma^{\text {in }}, \quad-\mathbf{b} \cdot \mathbf{n}_{\Gamma} u_{1}=\nu \frac{\partial u_{2}}{\partial \mathbf{n}_{\Gamma}}-\mathbf{b} \cdot \mathbf{n}_{\Gamma} u_{2} \quad \text { on } \Gamma^{\text {out }} .
$$

The last set of interface conditions is named IC2. Let $\mathbf{b} \in\left[W^{1, \infty}(\Omega)\right]^{d}$ and $\widetilde{\Omega}$ be either $\Omega_{1}$ or $\Omega_{2}$. Thanks to the assumption made on both $\Omega$ and $\Gamma, \widetilde{\Omega}$ has a Lipschitz continuous boundary, piecewise $C^{1,1}$.

Given an open subset $\Sigma \subseteq \partial \widetilde{\Omega}$ of class $C^{1,1}$, whose outward normal unit vector is denoted by $\mathbf{n}_{\Sigma}$, we define the following Hilbert spaces (see $[18,22]$ ) endowed with their standard norms:

$$
\begin{gathered}
L_{\mathbf{b}}^{2}(\Sigma)=\left\{v: \Sigma \rightarrow \mathbb{R}: \sqrt{\left|\mathbf{b} \cdot \mathbf{n}_{\Sigma}\right|} v \in L^{2}(\Sigma)\right\}, \quad\|v\|_{L_{\mathbf{b}}^{2}(\Sigma)}=\left(\int_{\Sigma}\left|\mathbf{b} \cdot \mathbf{n}_{\Sigma}\right| v^{2} d \Sigma\right)^{1 / 2}, \quad(2.12) \\
H_{00}^{1 / 2}(\Sigma)=\left\{v: L^{2}(\Sigma): \exists \tilde{v} \in H^{1 / 2}(\partial \widetilde{\Omega}):\left.\tilde{v}\right|_{\Sigma}=v,\left.\tilde{v}\right|_{\partial \widetilde{\Omega} \backslash \Sigma}=0\right\}, \\
\|v\|_{H_{00}^{1 / 2}(\Sigma)}=\inf _{v \in H^{1}(\widetilde{\Omega})}\|v\|_{H^{1}(\widetilde{\Omega})}, \\
L_{\operatorname{div}, \mathbf{b}}^{2}(\widetilde{\Omega})=\left\{v \in L^{2}(\widetilde{\Omega}), \operatorname{div}(\mathbf{b} v) \in L^{2}(\widetilde{\Omega})\right\}, \quad\|v\|_{L_{\text {div }, \mathbf{b}}^{2}(\widetilde{\Omega})}=\left(\|v\|_{L^{2}(\widetilde{\Omega})}^{2}+\|\operatorname{div}(\mathbf{b} v)\|_{L^{2}(\widetilde{\Omega})}^{2}\right)^{1 / 2},
\end{gathered}
$$

and finally

$$
X_{\mathbf{b}}(\widetilde{\Omega})=\left\{v \in L_{\mathrm{div}, \mathbf{b}}^{2}(\widetilde{\Omega}): v \in L_{\mathbf{b}}^{2}(\partial \widetilde{\Omega})\right\}, \quad\|v\|_{X_{\mathbf{b}}(\widetilde{\Omega})}=\left(\|v\|_{L_{\mathrm{div}, \mathbf{b}}^{2}(\widetilde{\Omega})}^{2}+\|v\|_{L_{\mathbf{b}}^{2}(\partial \widetilde{\Omega})}^{2}\right)^{1 / 2} .
$$

The following result has been proved in [18].

Theorem 2.1. Assume the following regularity properties on the data:

$\partial \Omega_{1}$ and $\partial \Omega_{2}$ are Lipschitz continuous, piecewise $C^{1,1} ; \quad \Gamma$ is of class $C^{1,1}$;

$$
\nu \in L^{\infty}\left(\Omega_{2}\right), \quad \mathbf{b} \in\left[W^{1, \infty}(\Omega)\right]^{d}, \quad b_{0} \in L^{\infty}(\Omega), \quad f \in L^{2}(\Omega) .
$$

Finally, assume that (2.3) holds. Then there is a unique solution $\left(u_{1}, u_{2}\right) \in L^{2}\left(\Omega_{1}\right) \times H^{1}\left(\Omega_{2}\right)$ of (2.10), where: equations (2.10) $)_{1,2}$ hold in the sense of distributions in $\Omega_{1}$ and $\Omega_{2}$, respectively; boundary condition (2.10) ${ }_{3}$ holds a.e. on $\left(\partial \Omega_{1} \backslash \Gamma\right)^{\text {in }}$; boundary condition (2.10) ${ }_{4}$ holds in $H^{1 / 2}\left(\partial \Omega_{2}\right)$; interface condition (2.10) 5 holds a.e. on $\Gamma^{\text {in }}$, interface condition (2.10) 6 holds in $\left(H_{00}^{1 / 2}(\Gamma)\right)^{\prime}$. Finally, the heterogeneous problem (2.10) can be regarded as the limit of a family of globally elliptic variational problems. 
Remark 2.1. Other interface conditions have been proposed in the literature to close system (2.4), (2.5), (2.8). For instance, (see [11])

$$
-\mathbf{b} \cdot \mathbf{n}_{\Gamma} u_{1}=\nu \frac{\partial u_{2}}{\partial \mathbf{n}_{\Gamma}}-\mathbf{b} \cdot \mathbf{n}_{\Gamma} u_{2} \quad \text { on } \Gamma^{\text {out }}, \quad u_{1}=u_{2}, \quad \frac{\partial u_{1}}{\partial \mathbf{n}_{\Gamma}}=\frac{\partial u_{2}}{\partial \mathbf{n}_{\Gamma}} \quad \text { on } \Gamma^{\text {in }},
$$

which are based on absorbing boundary condition theory, or else (see $[14,15])$

$$
u_{1}=u_{2} \quad \text { on } \Gamma, \quad \frac{\partial u_{1}}{\partial \mathbf{n}_{\Gamma}}=\frac{\partial u_{2}}{\partial \mathbf{n}_{\Gamma}} \quad \text { on } \Gamma^{i n} .
$$

However, the coupled problem with either one of these sets of conditions ((2.18), (2.19)) cannot be regarded as a limit of the same variational problem as $\nu \rightarrow 0$ in $\Omega_{1}$. For a survey on this subject we refer to $[16,10,6]$ for $1 \mathrm{D}$ problems, to [19] for $2 \mathrm{D}$ problems with convection limited to only one coordinate direction, and to $[11,14,15]$ for $2 \mathrm{D}$ problems.

In the next Sections we will consider another possible approach to close the heterogeneous problem (2.4), (2.5), (2.8) based on a saddle-point formulation of the coupled problem $([3,4])$.

The following results will be useful later. (We refer to [18] and [21] for their proof.)

Theorem 2.2. Under the same assumptions on the data as in Theorem 2.1, if $\lambda \in$ $H^{-1 / 2}\left(\partial \Omega_{1}\right)$ such that $\left.\lambda\right|_{\partial \Omega_{1}^{i n}} \in L_{\mathbf{b}}^{2}\left(\partial \Omega_{1}^{i n}\right)$, the first-order problem

$$
A_{1} u_{1}=f \quad \text { in } \Omega_{1}, \quad u_{1}=\lambda \quad \text { on }\left(\partial \Omega_{1}\right)^{\text {in }}
$$

admits a unique solution $u_{1} \in X_{\mathbf{b}}\left(\Omega_{1}\right)$.

If the Dirichlet condition (2.20) 2 is replaced by a flux condition

$$
\mathbf{b} \cdot \mathbf{n}_{1} u_{1}=\phi \quad \text { on }\left(\partial \Omega_{1}\right)^{\text {in }}
$$

with $\phi \in\left(H_{00}^{1 / 2}\left(\partial \Omega_{1}^{i n}\right)\right)^{\prime}$, the first-order problem (2.20) 1 , (2.21) is still well-posed.

Theorem 2.3. Under the same assumptions of Theorem 2.1,

1. if $\lambda \in H_{00}^{1 / 2}(\Gamma)$, the second-order problem

$$
A_{2} u_{2}=f \quad \text { in } \Omega_{2}, \quad u_{2}=0 \quad \text { on } \partial \Omega_{2} \backslash \Gamma, \quad u_{2}=\lambda \quad \text { on } \Gamma
$$

admits a unique solution in $H^{1}\left(\Omega_{2}\right)$;

2. if $\mu \in\left(H_{00}^{1 / 2}(\Gamma)\right)^{\prime}$, with $\mu_{\mid \Gamma^{o u t}} \in L_{\mathbf{b}}^{2}\left(\Gamma^{\text {out }}\right)$, the second-order problem

$$
\left\{\begin{array}{llll}
A_{2} u_{2}=f & \text { in } \Omega_{2}, & u_{2}=0 \quad \text { on } \partial \Omega_{2} \backslash \Gamma, & \\
\nu \frac{\partial u_{2}}{\partial \mathbf{n}_{2}}=0 & \text { on } \Gamma^{\text {in }}, & \nu \frac{\partial u_{2}}{\partial \mathbf{n}_{2}}-\mathbf{b} \cdot \mathbf{n}_{2} u_{2}=\mu & \text { on } \Gamma^{\text {out }}
\end{array}\right.
$$

admits a unique solution in $H^{1}\left(\Omega_{2}\right)$;

3. if $\mu \in\left(H_{00}^{1 / 2}(\Gamma)\right)^{\prime}$, and

$$
\|\mathbf{b}\|_{L^{\infty}(\Gamma)} \leqslant \varepsilon_{0}, \quad 0 \leqslant \varepsilon_{0} \leqslant \frac{2 \min \left\{\nu_{0}, \sigma_{0}\right\}}{C_{*}^{2}}
$$

(where $C_{*}$ is the constant of the trace inequality $\|v\|_{L^{2}\left(\partial \Omega_{2}\right)} \leqslant C_{*}\|v\|_{H^{1}\left(\Omega_{2}\right)}, \forall v \in$ $H^{1}\left(\Omega_{2}\right)$ ), the second-order problem

$$
A_{2} u_{2}=f \quad \text { in } \Omega_{2}, \quad u_{2}=0 \quad \text { on } \partial \Omega_{2} \backslash \Gamma, \quad \nu \frac{\partial u_{2}}{\partial \mathbf{n}_{2}}-\mathbf{b} \cdot \mathbf{n}_{2} u_{2}=\mu \quad \text { on } \Gamma(2.25)
$$

admits a unique solution in $H^{1}\left(\Omega_{2}\right)$. 


\section{Extended Variational Formulation (XVF)}

In this Section, we reformulate the coupled advection/advection-diffusion problem by regarding the continuity across $\Gamma^{i n}$ as a constraint, yielding a saddle-point problem.

Let the regularity assumptions (2.3) and (2.16)-(2.17) hold on the data.

Let us consider the Hilbert spaces $L_{\mathbf{b}}^{2}\left(\Gamma^{i n}\right)$ and $X_{\mathbf{b}}\left(\Omega_{1}\right)$ defined in (2.12) and (2.15), respectively. Moreover we define

$$
\Lambda_{1}=L_{\mathbf{b}}^{2}\left(\Gamma^{i n}\right), \quad \Lambda_{2}=H_{00}^{1 / 2}(\Gamma), \quad V_{1}=X_{\mathbf{b}}\left(\Omega_{1}\right), \quad V_{2}=H^{1}\left(\Omega_{2}\right), \quad V=V_{1} \times V_{2} .
$$

The space $V$, endowed with the graph norm $\|v\|_{V}=\left(\left\|v_{1}\right\|_{X_{\mathbf{b}}\left(\Omega_{1}\right)}^{2}+\left\|v_{2}\right\|_{H^{1}\left(\Omega_{2}\right)}^{2}\right)^{1 / 2}$, is a Hilbert space, then we set

$$
\begin{gathered}
V_{1}^{0}=\left\{v_{1} \in X_{\mathbf{b}}\left(\Omega_{1}\right), v_{1}=0 \text { a.e. in }\left(\partial \Omega_{1} \backslash \Gamma\right)^{i n}\right\} \subset V_{1}, \\
V_{2}^{0}=\left\{v_{2} \in H^{1}\left(\Omega_{2}\right): v_{2 \mid\left(\partial \Omega_{2} \backslash \Gamma\right)}=0\right\} \subset V_{2}
\end{gathered}
$$

and $V^{0}=V_{1}^{0} \times V_{2}^{0}$. We introduce the bilinear form: $a: V^{0} \times V^{0} \rightarrow \mathbb{R}$ :

$$
\begin{aligned}
a(u, v)= & \int_{\Omega_{1}} \operatorname{div}\left(\mathbf{b} u_{1}\right) v_{1} d \Omega+\int_{\Omega_{1}} b_{0} u_{1} v_{1} d \Omega-\int_{\Gamma^{\text {in }}} \mathbf{b} \cdot \mathbf{n}_{\Gamma} u_{\alpha} v_{\alpha} d \Gamma \\
& +\int_{\Omega_{2}} \nu \nabla u_{2} \cdot \nabla v_{2} d \Omega-\int_{\Omega_{2}} u_{2} \mathbf{b} \cdot \nabla v_{2} d \Omega+\int_{\Omega_{2}} b_{0} u_{2} v_{2} d \Omega \\
& -\int_{\Gamma_{\text {out }}} \mathbf{b} \cdot \mathbf{n}_{\Gamma} u_{1} v_{2} d \Gamma
\end{aligned}
$$

where $u_{\alpha}=\alpha u_{1}+(1-\alpha) u_{2}, v_{\alpha}=\alpha v_{1}+(1-\alpha) v_{2}, \alpha$ can be either 0 or 1 , and the linear continuous functional $F: V^{0} \rightarrow \mathbb{R}$ :

$$
F(v)=\int_{\Omega_{1}} f v_{1} d \Omega+\int_{\Omega_{2}} f v_{2} d \Omega .
$$

As we will see in the proof of the next Theorem, the choice of the parameter $\alpha$ is responsible for the setting of interface conditions across the inflow interface, more precisely, $\alpha=1$ ( $\alpha=0$, resp.) will provide interface conditions (2.9) ((2.11), resp.).

The bilinear form $a$ is continuous. Let us bound ourselves to check only the integrals on the interface. Since $u, v \in V^{0}$, then $u_{1}, v_{1} \in L_{\mathbf{b}}^{2}\left(\Gamma^{i n}\right)$ and $\int_{\Gamma^{i n}} \mathbf{b} \cdot \mathbf{n}_{\Gamma} u_{1} v_{1} d \Gamma$ is therefore bounded. For the regularity assumptions $(2.17)$ on $\mathbf{b}$, it holds $H^{1 / 2}(\Gamma) \subset L_{\mathbf{b}}^{2}(\Gamma)$, so that also the boundedness of the mixed integral $\int_{\Gamma^{\text {out }}} \mathbf{b} \cdot \mathbf{n}_{\Gamma} u_{1} v_{2} d \Gamma$ is guaranteed. Finally the integral $\int_{\Gamma^{i n}} \mathbf{b} \cdot \mathbf{n}_{\Gamma} u_{2} v_{2} d \Gamma$ can be interpreted as a duality pair between $H_{00}^{1 / 2}(\Gamma)$ and its dual space. We denote by $s$ an element in $\Lambda_{1}^{\prime}$. Note that the dual space of $\Lambda_{1}$ is

$$
\Lambda_{1}^{\prime}=L_{1 / \mathbf{b}}^{2}\left(\Gamma^{i n}\right)=\left\{s: \Gamma^{i n} \rightarrow \mathbb{R}:\left(\left|\mathbf{b} \cdot \mathbf{n}_{\Gamma}\right|\right)^{-1 / 2} s \in L^{2}\left(\Gamma^{i n}\right)\right\}
$$

and the duality between $\Lambda_{1}$ and $\Lambda_{1}^{\prime}$ can be written as

$$
\Lambda_{1}^{\prime}\langle s, \phi\rangle_{\Lambda_{1}}=\int_{\Gamma^{i n}} s \phi d \Gamma .
$$


Finally, let us define the following bilinear continuous form:

$$
b: V^{0} \times \Lambda_{1}^{\prime} \rightarrow \mathbb{R}: \quad b(v, s)={ }_{\Lambda_{1}^{\prime}}\left\langle s,\left(v_{1}-v_{2}\right)_{\mid \Gamma^{i n}}\right\rangle_{\Lambda_{1}}
$$

Note that $\left(v_{1}-v_{2}\right)_{\mid \Gamma^{i n}}$ is well defined and it belongs to $L_{\mathbf{b}}^{2}\left(\Gamma^{i n}\right)$. As a matter of fact, $v_{2 \mid \Gamma} \in H_{00}^{1 / 2}(\Gamma)$ and, since regularity assumptions $(2.16)-(2.17)$ hold, its restriction to $\Gamma^{i n}$ belongs to $L_{\mathbf{b}}^{2}\left(\Gamma^{i n}\right)$. Then we set

$$
Z=\left\{v \in V^{0}: b(v, s)=0, \forall s \in \Lambda_{1}^{\prime}\right\}
$$

since $b$ is continuous on $V^{0}$, then $Z$ is a closed subspace of $V^{0}$ and then it is a Hilbert space with respect to the norm of $V$.

We are now able to define the saddle-point problem

$$
\text { seek } u \in V^{0}, t \in \Lambda_{1}^{\prime}: \begin{cases}a(u, v)+b(v, t)=F(v) & \forall v \in V^{0} \\ b(u, s)=0 & \forall s \in \Lambda_{1}^{\prime} .\end{cases}
$$

\subsection{Well-posedness and Euler-Lagrange equations}

Our aim is twofold. From one hand we want to prove the well-posedness of the saddle-point problem (3.9). On the other hand we want to characterize the multiplier $t \in \Lambda_{1}^{\prime}$ and recover the interface conditions on $\Gamma$ that are hidden in this formulation.

We begin by defining the following linear and continuous operators:

$$
\begin{aligned}
& A: V^{0} \rightarrow\left(V^{0}\right)^{\prime} \quad V^{\prime}\langle A u, v\rangle_{V}=a(u, v) \quad \forall u, v \in V^{0}, \\
& B: V^{0} \rightarrow \Lambda_{1} \quad \Lambda_{1}^{\prime}\langle s, B v\rangle_{\Lambda_{1}}=b(v, s) \quad \forall v \in V^{0}, \forall s \in \Lambda_{1}^{\prime} .
\end{aligned}
$$

By definition (3.8), we have $B v=\left(v_{1}-v_{2}\right)_{\mid \Gamma^{i n}}$, and $Z=\operatorname{ker}(B) \subset V^{0}$, i.e.,

$$
Z=\left\{v \in V^{0}: v_{1}=v_{2} \text { a.e. on } \Gamma^{i n}\right\} \text {. }
$$

We now introduce the orthogonal of $Z: Z^{\perp}=\left\{v \in V^{0}:(v, z)=0 \forall z \in Z\right\}$, where $(\cdot, \cdot)$ denotes the inner product on $V$, and the so called polar set of $Z^{\perp}:\left(Z^{\perp}\right)^{\circ}=\left\{f \in\left(V^{0}\right)^{\prime}\right.$ : $\left.V^{\prime}\langle f, v\rangle_{V}=0 \forall v \in Z^{\perp}\right\}$. The dual space $Z^{\prime}$ of $Z$ can be identified with $\left(Z^{\perp}\right)^{\circ}$ (see [7, 12]), the latter being a closed subspace of $\left(V^{0}\right)^{\prime}$. Finally, we define the linear and continuous operator

$$
\pi A: Z \rightarrow Z^{\prime}: \quad{ }^{\prime}\langle\pi A u, v\rangle_{V}={ }_{V^{\prime}}\langle A u, v\rangle_{V}, \quad \forall u, v \in Z,
$$

where $\pi:\left(V^{0}\right)^{\prime} \rightarrow Z^{\prime}$ is the orthogonal projection from $\left(V^{0}\right)^{\prime}$ onto $Z^{\prime}$.

The following theorem states the well-posedness of the saddle-point problem (3.9).

Theorem 3.1. If regularity assumptions (2.3) and (2.16)-(2.17) hold on the data and $\mathbf{b}$ satisfies the smallness assumption (2.24), then there exists a unique solution $(u, t) \in V^{0} \times \Lambda_{1}^{\prime}$ of (3.9) and the solution $u=\left(u_{1}, u_{2}\right)$ satisfies the interface conditions (2.9) ((2.11), resp.) when $\alpha=1$ ( $\alpha=0$, resp.).

Proof. We have seen above that $V^{0}$ and $\Lambda_{1}^{\prime}$ are Hilbert spaces, the bilinear forms $a$ and $b$ are continuous and $F \in\left(V^{0}\right)^{\prime}$. Thus, existence and uniqueness of solution $(u, t)$ of $(3.9)$ are ensured if (see [7]): 
i) $\pi A$ is an isomorphism from $Z$ onto $Z^{\prime}$,

ii) $\exists \beta>0$ such that

$$
\inf _{s \in \Lambda_{1}^{\prime}} \sup _{v \in V^{0}} \frac{b(v, s)}{\|v\|_{V}\|s\|_{\Lambda_{1}^{\prime}}} \geqslant \beta .
$$

Proof of $i$ ). Thanks to the Banach-Nečas-Babuska theorem (see, e.g., [13]), $\pi A$ is an isomorphism from $Z$ onto $Z^{\prime}$ iff

$$
\begin{array}{r}
\exists C_{0}>0:\|\pi A u\|_{V^{\prime}} \geqslant C_{0}\|u\|_{V} \quad \forall u \in Z \\
\forall w \in Z \quad(a(w, v)=0 \quad \forall v \in Z) \Rightarrow w=0 .
\end{array}
$$

Condition (3.11) is often referred to by saying that $\pi A$ is bounding ([23, Thm 4.15]) and it is equivalent to prove that $\pi A$ is injective and its range is closed in $Z^{\prime}$, while condition (3.12) means that the adjoint operator $(\pi A)^{*}$ of $(\pi A)$ is injective.

Let us start by proving that $\pi A$ is bounding. For any $v \in Z$, it holds $v_{\alpha}=v_{1}=v_{2}$ and

$$
\begin{aligned}
V^{\prime}\langle\pi A u, u\rangle_{V}= & a(u, u)=\int_{\Omega_{1}}\left(\frac{1}{2} \operatorname{div} \mathbf{b}+b_{0}\right) u_{1}^{2} d \Omega+\frac{1}{2} \int_{\partial \Omega_{1}} \mathbf{b} \cdot \mathbf{n}_{1} u_{1}^{2} d \Gamma-\int_{\Gamma^{\text {in }}} \mathbf{b} \cdot \mathbf{n}_{\Gamma} u_{1}^{2} d \Gamma \\
& +\int_{\Omega_{2}} \nu\left|\nabla u_{2}\right|^{2} d \Omega+\int_{\Omega_{2}}\left(\frac{1}{2} \operatorname{div} \mathbf{b}+b_{0}\right) u_{2}^{2} d \Omega-\frac{1}{2} \int_{\Gamma} \mathbf{b} \cdot \mathbf{n}_{2} u_{2}^{2} d \Gamma \\
& -\int_{\Gamma^{\text {out }}} \mathbf{b} \cdot \mathbf{n}_{\Gamma} u_{1} u_{2} d \Gamma \\
\geqslant & \sigma_{0}\left\|u_{1}\right\|_{L^{2}\left(\Omega_{1}\right)}^{2}+\min \left\{\nu_{0}, \sigma_{0}\right\}\left\|u_{2}\right\|_{H^{1}\left(\Omega_{2}\right)}^{2}+\frac{1}{2} \int_{\left(\partial \Omega_{1} \backslash \Gamma\right)^{\text {out }}} \mathbf{b} \cdot \mathbf{n}_{1} u_{1}^{2} d \Gamma \\
& +\frac{1}{2} \int_{\Gamma^{\text {out }}} \mathbf{b} \cdot \mathbf{n}_{\Gamma} u_{1}^{2} d \Gamma-\frac{1}{2} \int_{\Gamma^{\text {in }}} \mathbf{b} \cdot \mathbf{n}_{\Gamma} u_{1}^{2} d \Gamma-\frac{1}{2} \int_{\Gamma^{\text {in }}} \mathbf{b} \cdot \mathbf{n}_{2} u_{2}^{2} d \Gamma \\
& -\frac{1}{2} \int_{\Gamma^{\text {out }}} \mathbf{b} \cdot \mathbf{n}_{2} u_{2}^{2} d \Gamma-\int_{\Gamma^{\text {out }}} \mathbf{b} \cdot \mathbf{n}_{\Gamma} u_{1} u_{2} d \Gamma \\
= & \sigma_{0}\left\|u_{1}\right\|_{L^{2}\left(\Omega_{1}\right)}^{2}+\min \left\{\nu_{0}, \sigma_{0}\right\}\left\|u_{2}\right\|_{H^{1}\left(\Omega_{2}\right)}^{2} \\
& +\frac{1}{2} \int_{\left(\partial \Omega_{1} \backslash \Gamma\right)^{\text {out }}} \mathbf{b} \cdot \mathbf{n}_{1} u_{1}^{2}+\frac{1}{2} \int_{\Gamma^{\text {out }}} \mathbf{b} \cdot \mathbf{n}_{\Gamma}\left(u_{1}-u_{2}\right)^{2} d \Gamma \\
\geqslant & \sigma_{0}\left\|u_{1}\right\|_{L^{2}\left(\Omega_{1}\right)}^{2}+\min \left\{\nu_{0}, \sigma_{0}\right\}\left\|u_{2}\right\|_{H^{1}\left(\Omega_{2}\right)}^{2} \\
& +\frac{1}{4}\left(\left\|u_{1}\right\|_{L_{\mathbf{b}}^{2}\left(\partial \Omega_{1}\right)}^{2}-\int_{\Gamma}\left|\mathbf{b} \cdot \mathbf{n}_{\Gamma}\right| u_{2}^{2} d \Gamma\right)
\end{aligned}
$$

where we have used the fact that $u_{1}=u_{2}$ on $\Gamma^{\text {in }}$ and $\left(u_{1}-u_{2}\right)^{2} \geqslant(1-\varepsilon) u_{1}^{2}+(1-1 / \varepsilon) u_{2}^{2}$ with $\varepsilon=1 / 2$. By the trace inequality $\left\|u_{2}\right\|_{L^{2}(\Gamma)} \leqslant C_{*}\left\|u_{2}\right\|_{H^{1}\left(\Omega_{2}\right)}$, it holds $\left\|u_{2}\right\|_{L_{\mathbf{b}}^{2}(\Gamma)}^{2} \leqslant$ $C_{*}^{2}\|\mathbf{b}\|_{L^{\infty}(\Gamma)}\left\|u_{2}\right\|_{H^{1}\left(\Omega_{2}\right)}^{2}$ and, under assumption (2.24) we define the positive constant $C_{1}=$ $\min \left\{\nu_{0}, \sigma_{0}\right\}-C_{*}^{2}\|\mathbf{b}\|_{L^{\infty}(\Gamma)}$, so that

$$
{ }_{V^{\prime}}\langle\pi A u, u\rangle_{V} \geqslant \sigma_{0}\left\|u_{1}\right\|_{L^{2}\left(\Omega_{1}\right)}^{2}+C_{1}\left\|u_{2}\right\|_{H^{1}\left(\Omega_{2}\right)}^{2}+\frac{1}{4}\left\|u_{1}\right\|_{L_{\mathbf{b}}^{2}\left(\partial \Omega_{1}\right)}^{2}
$$

For any $v=\left(v_{1}, v_{2}\right) \in V$ we define the norm

$$
\|v\|_{L}=\left(\left\|v_{1}\right\|_{L^{2}\left(\Omega_{1}\right)}^{2}+\left\|v_{2}\right\|_{H^{1}\left(\Omega_{2}\right)}^{2}+\left\|v_{1}\right\|_{L_{\mathbf{b}}^{2}\left(\partial \Omega_{1}\right)}^{2}\right)^{1 / 2} .
$$


It is straightforward to prove that $\exists M>0$ such that $|a(u, v)| \leqslant M\|u\|_{V}\|v\|_{L}$ for any $u, v \in V^{0}$, while (3.13) says that

$$
\exists C_{2}>0: \quad a(u, u) \geqslant C_{2}\|u\|_{L}^{2} \quad \forall u \in Z .
$$

We set $\tilde{a}(u, v)=a(u, v)-\left(\operatorname{div}\left(\mathbf{b} u_{1}\right), v_{1}\right)_{L^{2}\left(\Omega_{1}\right)}$, therefore there exists $\tilde{M}>0$ such that $|\tilde{a}(u, v)| \leqslant \tilde{M}\|u\|_{L}\|v\|_{L}$ for any $u, v \in V^{0}$. Since $\|v\|_{L} \leqslant\|v\|_{V}$ for any $v \in V^{0}$, it holds

$$
\begin{aligned}
\sup _{v \in Z} \frac{a(u, v)}{\|v\|_{V}} & \geqslant \sup _{v \in Z} \frac{a(u, v)}{\|v\|_{L}} \geqslant \sup _{v \in Z} \frac{\left(\operatorname{div}\left(\mathbf{b} u_{1}\right), v_{1}\right)_{L^{2}\left(\Omega_{1}\right)}}{\|v\|_{L}}-\tilde{M}\|u\|_{L} \\
& \geqslant \sup _{v_{1} \in V_{1}} \frac{\left(\operatorname{div}\left(\mathbf{b} u_{1}\right), v_{1}\right)_{L^{2}(\Omega)}}{\left\|v_{1}\right\|_{V_{1}}}-\frac{\tilde{M}}{C_{2}} \sup _{v \in Z} \frac{a(u, v)}{\|v\|_{L}}
\end{aligned}
$$

so that

$$
\left(1+\frac{\tilde{M}}{C_{2}}+\frac{1}{C_{2}}\right) \sup _{v \in Z} \frac{a(u, v)}{\|v\|_{V}} \geqslant\left\|\operatorname{div}\left(\mathbf{b} u_{1}\right)\right\|_{L^{2}(\Omega)}+\|u\|_{L} \geqslant C_{3}\|u\|_{V},
$$

where $C_{3}>0$. (3.11) follows with $C_{0}=C_{2} C_{3} /\left(C_{2}+\tilde{M}+1\right)$.

In order to prove (3.12), we start by noting that $a(w, v)=0$ for any $v \in Z$, then we take $v=w$. By applying (3.15) we conclude that $w=0$ a.e. in $\Omega$.

Proof of ii). As a consequence of the Closed Range theorem ([25]) together with the Open Mapping Theorem, the inf-sup condition (3.10) is satisfied iff the operator $B$ is surjective from $V$ onto $\Lambda_{1}$.

For the regularity assumption $(2.16)-(2.17)$, it holds that $H_{00}^{1 / 2}\left(\Gamma^{i n}\right) \subset L_{\mathbf{b}}^{2}\left(\Gamma^{\text {in }}\right)$. Let $\lambda \in L_{\mathbf{b}}^{2}\left(\Gamma^{i n}\right)$, for any $\lambda_{2} \in H_{00}^{1 / 2}\left(\Gamma^{i n}\right)$ we can define $\lambda_{1} \in L_{\mathbf{b}}^{2}\left(\Gamma^{i n}\right)$ such that $\lambda_{1}=\lambda+\lambda_{2}$, solve the differential problems

$$
\left\{\begin{array} { l l } 
{ A _ { 1 } v _ { 1 } = 0 } & { \text { in } \Omega _ { 1 } } \\
{ v _ { 1 } = 0 } & { \text { a.e. on } ( \partial \Omega _ { 1 } \backslash \Gamma ) ^ { i n } } \\
{ v _ { 1 } = \lambda _ { 1 } } & { \text { a.e. on } \Gamma ^ { \text { in } } }
\end{array} \quad \left\{\begin{array}{ll}
A_{2} v_{2}=0 & \text { in } \Omega_{2} \\
v_{2}=0 & \text { on } \partial \Omega_{2} \backslash \Gamma^{\text {in }} \\
v_{2}=\lambda_{2} & \text { on } \Gamma^{\text {in }}
\end{array}\right.\right.
$$

and define $v=\left(v_{1}, v_{2}\right)$. By Theorems 2.2, 2.3 it holds $v \in V^{0}$ and $B v=\left(v_{1}-v_{2}\right)_{\mid \Gamma^{\text {in }}}=$ $\lambda_{1}-\lambda_{2}=\lambda$, i.e., $B$ is surjective and the saddle-point problem (3.9) is well-posed.

It remains to prove that the solution $u=\left(u_{1}, u_{2}\right)$ of (3.9) satisfies the interface conditions (2.9). To this aim, we integrate by parts the first equation of (3.9). It holds

$$
\begin{aligned}
a(u, v)+b(v, t)= & \int_{\Omega_{1}}\left(A_{1} u_{1}\right) v_{1} d \Omega-\int_{\Gamma^{\text {in }}} \mathbf{b} \cdot \mathbf{n}_{\Gamma} u_{\alpha} v_{\alpha} d \Gamma \\
& +\int_{\Omega_{2}}\left(A_{2} u_{2}\right) v_{2} d \Omega-\int_{\Gamma}\left(\nu \frac{\partial u_{2}}{\partial n_{\Gamma}}-\mathbf{b} \cdot \mathbf{n}_{\Gamma} u_{2}\right) v_{2} d \Gamma \\
& -\int_{\Gamma^{\text {out }}} \mathbf{b} \cdot \mathbf{n}_{\Gamma} u_{1} v_{2} d \Gamma+\Lambda_{1}^{\prime}\left\langle t,\left(v_{1}-v_{2}\right)_{\mid \Gamma^{\text {in }}}\right\rangle_{\Lambda_{1}} \\
= & \int_{\Omega_{1}} f v_{1} d \Omega+\int_{\Omega_{2}} f v_{2} d \Omega \quad \forall v \in V^{0}
\end{aligned}
$$


By taking $v_{1} \in C_{0}^{\infty}\left(\Omega_{1}\right), v_{2}=0$ first, then $v_{1}=0, v_{2} \in C_{0}^{\infty}\left(\Omega_{2}\right)$, and

$$
A_{1} u_{1}=f \quad \text { a.e. in } \Omega_{1}, \quad A_{2} u_{2}=f \quad \text { a.e. in } \Omega_{2} \text {. }
$$

Let us take now $v=0$ and $s_{1} \in \Lambda_{1}^{\prime}$, from the second equation of (3.9) it follows:

$$
u_{1}=u_{2} \text { a.e. on } \Gamma^{i n},
$$

that is the interface condition $(2.9)_{1}$. Finally, starting from $(3.17)$, for any $v \in V^{0},(3.18)$ implies

$$
\begin{aligned}
-\int_{\Gamma^{\text {in }}} \mathbf{b} \cdot \mathbf{n}_{\Gamma} u_{\alpha} v_{\alpha} d \Gamma-\int_{\Gamma}\left(\nu \frac{\partial u_{2}}{\partial n_{\Gamma}}-\mathbf{b} \cdot \mathbf{n}_{\Gamma} u_{2}\right) v_{2} d \Gamma & \\
- & \int_{\Gamma^{\text {out }}} \mathbf{b} \cdot \mathbf{n}_{\Gamma} u_{1} v_{2} d \Gamma+{ }_{\Lambda_{1}^{\prime}}\left\langle t,\left(v_{1}-v_{2}\right)_{\mid \Gamma^{\text {in }}}\right\rangle_{\Lambda_{1}}=0 \quad \forall v \in V^{0} .
\end{aligned}
$$

The exploitation of the integral on $\Gamma$ as the sum of integrals on $\Gamma^{i n}$ and $\Gamma^{\text {out }}$ leads to

$$
\begin{aligned}
& -\int_{\Gamma^{i n}} \mathbf{b} \cdot \mathbf{n}_{\Gamma} u_{\alpha} v_{\alpha} d \Gamma-\int_{\Gamma^{i n}}\left(\nu \frac{\partial u_{2}}{\partial n_{\Gamma}}-\mathbf{b} \cdot \mathbf{n}_{\Gamma} u_{2}\right) v_{2} d \Gamma \\
& { }_{\Lambda_{1}^{\prime}}\left\langle t,\left(v_{1}-v_{2}\right)_{\mid \Gamma^{i n}}\right\rangle_{\Lambda_{1}}-\int_{\Gamma^{\text {out }}}\left(\nu \frac{\partial u_{2}}{\partial n_{\Gamma}}-\mathbf{b} \cdot \mathbf{n}_{\Gamma} u_{2}+\mathbf{b} \cdot \mathbf{n}_{\Gamma} u_{1}\right) v_{2} d \Gamma=0 \quad \forall v \in V^{0} .
\end{aligned}
$$

The interface condition on $\Gamma^{\text {out }}$ easily reads as

$$
\mathbf{b} \cdot \mathbf{n}_{\Gamma} u_{1}+\nu \frac{\partial u_{2}}{\partial n_{\Gamma}}-\mathbf{b} \cdot \mathbf{n}_{\Gamma} u_{2}=0 \quad \text { in }\left(H_{00}^{1 / 2}\left(\Gamma^{o u t}\right)\right)^{\prime},
$$

while the characterization of $t \in \Lambda_{1}^{\prime}$ depends on $\alpha$ as well as the interface condition on $\Gamma^{i n}$. When $\alpha=1$ the multiplier $t$ is

$$
t=\mathbf{b} \cdot \mathbf{n}_{\Gamma} u_{1}=-\nu \frac{\partial u_{2}}{\partial n_{\Gamma}}+\mathbf{b} \cdot \mathbf{n}_{\Gamma} u_{2}
$$

and the corresponding interface condition on $\Gamma^{i n}$ reads

$$
-\nu \frac{\partial u_{2}}{\partial n_{\Gamma}}+\mathbf{b} \cdot \mathbf{n}_{\Gamma} u_{2}-\mathbf{b} \cdot \mathbf{n}_{\Gamma} u_{1}=0 \quad \text { in }\left(H_{00}^{1 / 2}\left(\Gamma^{i n}\right)\right)^{\prime},
$$

while when $\alpha=0$ the multiplier $t$ is

$$
t=0=-\nu \frac{\partial u_{2}}{\partial n_{\Gamma}}
$$

and the corresponding interface condition on $\Gamma^{\text {in }}$ reads

$$
-\nu \frac{\partial u_{2}}{\partial n_{\Gamma}}=0 \quad \text { in }\left(H_{00}^{1 / 2}\left(\Gamma^{i n}\right)\right)^{\prime} .
$$

Therefore, when $\alpha=1$ we obtain the interface conditions IC1 (2.9), while when $\alpha=0$ we recover interface conditions IC2 (2.11). In view of (3.19) and the characterization of $t$, it holds $\left.u_{1}\right|_{\Gamma^{\text {in }}} \in H^{1 / 2}\left(\Gamma^{\text {in }}\right)$ and $t \in\left(H_{00}^{1 / 2}(\Gamma)\right)^{\prime}$. 
From the previous theorem we deduce that the saddle-point problem (3.9) is equivalent to the heterogeneous problem (2.10).

Remark 3.1. It is important to note that the interface condition $u_{1}=u_{2}$ a.e. on $\Gamma^{i n}$, cannot be extended to the whole interface $\Gamma$ since it would induce the definition of a linear operator $B$

$$
B: V^{0} \rightarrow L_{\mathbf{b}}^{2}(\Gamma): \quad B v=\left(v_{1}-v_{2}\right)_{\mid \Gamma},
$$

which is not surjective. As a matter of fact, if $\Gamma^{o u t} \neq \emptyset$, it is not guaranteed that, given a function $\lambda_{1} \in L_{\mathbf{b}}^{2}(\Gamma)$, there exists a function $v_{1} \in V_{1}^{0}$ such that its restriction to $\Gamma$ coincides with $\lambda_{1}$.

\section{The interface problem}

In Sections 2-3 we have shown the equivalence at continuous level between the heterogeneous formulation (2.10) of Gastaldi et al. [18] and the extended variational formulation (3.9), by proving that both formulations provide the same set of interface conditions.

In Section 4.1 we recall the Steklov-Poincaré equation associated to (2.10), see [18], while in Section 4.2, starting from the saddle-point formulation (3.9), we derive the associated interface equation by setting the decomposition of $u_{1}$ and $u_{2}$ and their variations, introducing the interface variables and unveiling the interface balance equations.

\subsection{Steklov-Poincaré equation for the heterogeneous problem (2.10)}

For $k=1,2$, we write each $u_{k}$ as the sum of two functions, the former $u_{k}^{\lambda_{k}}$ depending on the unknown trace $\lambda_{k}$ of $u_{k}$ at the interface $\Gamma$, the latter $u_{k}^{f}$ depending on the forcing term $f$, i.e.,

$$
u_{1}=u_{1}^{\lambda_{1}}+u_{1}^{f}, \quad u_{2}=u_{2}^{\lambda_{2}}+u_{2}^{f} .
$$

Thanks to the linearity of the differential problem, the corresponding test functions $v_{k}$ depend only on the trace $\mu_{k}$ on $\Gamma$ (not on the external force $f$ ), i.e., $v_{k}=v_{k}^{\mu_{k}}$ (for $k=1,2$ ). More precisely, $u_{1}^{f}$ and $u_{2}^{f}$ are the solutions of problems

$$
\left\{\begin{array} { l l } 
{ A _ { 1 } u _ { 1 } ^ { f } = f } & { \text { in } \Omega _ { 1 } } \\
{ u _ { 1 } ^ { f } = 0 } & { \text { on } ( \partial \Omega _ { 1 } ) ^ { i n } }
\end{array} \quad \left\{\begin{array}{ll}
A_{2} u_{2}^{f}=f & \text { in } \Omega_{2} \\
u_{2}^{f}=0 & \text { on } \partial \Omega_{2},
\end{array}\right.\right.
$$

while $u_{1}^{\lambda_{1}}$ and $u_{2}^{\lambda_{2}}$ are the solutions of

$$
\left\{\begin{array} { l l } 
{ A _ { 1 } u _ { 1 } ^ { \lambda _ { 1 } } = 0 } & { \text { in } \Omega _ { 1 } } \\
{ u _ { 1 } ^ { \lambda _ { 1 } } = 0 } & { \text { on } ( \partial \Omega _ { 1 } \backslash \Gamma ) ^ { i n } } \\
{ u _ { 1 } ^ { \lambda _ { 1 } } = \lambda _ { 1 } } & { \text { on } \Gamma ^ { \text { in } } , }
\end{array} \quad \left\{\begin{array}{ll}
A_{2} u_{2}^{\lambda_{2}}=0 & \text { in } \Omega_{2} \\
u_{2}^{\lambda_{2}}=0 & \text { on } \partial \Omega_{2} \backslash \Gamma \\
u_{2}^{\lambda_{2}}=\lambda_{2} & \text { on } \Gamma .
\end{array}\right.\right.
$$

For any $\lambda \in L^{2}(\Gamma)$, let $\lambda^{i n}=\left.\lambda\right|_{\Gamma^{i n}}$ denote the restriction of $\lambda$ to $\Gamma^{i n}$. Similarly, $v_{1}^{\mu_{1}}$ and $v_{2}^{\mu_{2}}$ are the solutions of problems (4.3) with data $\mu_{1}$ and $\mu_{2}$ instead of $\lambda_{1}$ and $\lambda_{2}$ on $\Gamma$, respectively.

We define the Steklov-Poincaré operators on the interface

$$
\begin{aligned}
\mathcal{S}_{1}: L_{\mathbf{b}}^{2}(\Gamma) \rightarrow & \left(H_{00}^{1 / 2}(\Gamma)\right)^{\prime}: \\
& \left\langle\left\langle\mathcal{S}_{1} \lambda_{1}, \mu_{2}\right\rangle\right\rangle_{\Gamma}=\int_{\Gamma}-\mathbf{b} \cdot \mathbf{n}_{1} u_{1}^{\lambda_{1}} \mu_{2} d \Gamma \quad \forall \mu_{2} \in H_{00}^{1 / 2}(\Gamma),
\end{aligned}
$$


(actually $\mathcal{S}_{1}$ depends only on $\lambda_{1}^{\text {in }}$ )

$\mathcal{S}_{2}: H_{00}^{1 / 2}(\Gamma) \rightarrow\left(H_{00}^{1 / 2}(\Gamma)\right)^{\prime}:$

$$
\left\langle\left\langle\mathcal{S}_{2} \lambda_{2}, \mu_{2}\right\rangle\right\rangle_{\Gamma}=a_{2}\left(u_{2}^{\lambda_{2}}, v_{2}^{\mu_{2}}\right)=\int_{\Gamma}\left(\nu \frac{\partial u_{2}^{\lambda_{2}}}{\partial \mathbf{n}_{2}}-\mathbf{b} \cdot \mathbf{n}_{2} u_{2}^{\lambda_{2}}\right) \mu_{2} d \Gamma,
$$

where for any subset $\Sigma \subseteq \Gamma,\left\langle\langle\cdot, \cdot\rangle_{\Gamma}\right.$ denotes the duality between $H_{00}^{1 / 2}(\Gamma)$ and $\left(H_{00}^{1 / 2}(\Gamma)\right)^{\prime}$.

System (2.10) can be equivalently written as seek $\lambda \in \Lambda_{2}=H_{00}^{1 / 2}(\Gamma)$ such that

$$
\langle\langle\mathcal{S} \lambda, \mu\rangle\rangle_{\Gamma}=\langle\langle\chi, \mu\rangle\rangle_{\Gamma} \quad \forall \mu \in \Lambda_{2}
$$

where

$$
\langle\langle\mathcal{S} \lambda, \mu\rangle\rangle_{\Gamma}=\left\langle\left\langle\mathcal{S}_{1} \lambda^{i n}, \mu\right\rangle\right\rangle_{\Gamma}+\left\langle\left\langle\mathcal{S}_{2} \lambda, \mu\right\rangle\right\rangle_{\Gamma}, \quad\langle\langle\chi, \mu\rangle\rangle_{\Gamma}=\left\langle\left\langle\chi_{1}, \mu\right\rangle\right\rangle_{\Gamma}+\left\langle\left\langle\chi_{2}, \mu\right\rangle\right\rangle_{\Gamma},
$$

$\lambda^{i n}=\left.\lambda\right|_{\Gamma^{i n}}$, while $\chi_{1}$ and $\chi_{2}$ are the fluxes on $\Gamma$ associated to $u_{1}^{f}$ and $u_{2}^{f}$, respectively, i.e.,

$$
\chi_{1}=\mathbf{b} \cdot \mathbf{n}_{1} u_{1}^{f}, \quad \chi_{2}=-\nu \frac{\partial u_{2}^{f}}{\partial \mathbf{n}_{2}}+\mathbf{b} \cdot \mathbf{n}_{2} u_{2}^{f}=-\nu \frac{\partial u_{2}^{f}}{\partial \mathbf{n}_{2}} .
$$

Note that $\left.\chi_{1}\right|_{\Gamma^{i n}}=0$.

We set the interface operators $\mathcal{S}_{1}^{0}: L_{\mathbf{b}}^{2}(\Gamma) \rightarrow\left(H_{00}^{1 / 2}(\Gamma)\right)^{\prime}$ and $\mathcal{S}_{2}^{0}: H_{00}^{1 / 2}(\Gamma) \rightarrow\left(H_{00}^{1 / 2}(\Gamma)\right)^{\prime}$ such that

$$
\mathcal{S}_{1}^{0} \lambda=\left\{\begin{array}{ll}
0 & \text { on } \Gamma^{\text {in }} \\
-\mathbf{b} \cdot \mathbf{n}_{1} u_{1}^{\lambda} & \text { on } \Gamma^{\text {out }},
\end{array} \quad \mathcal{S}_{2}^{0} \lambda= \begin{cases}\nu \frac{\partial u_{2}^{\lambda}}{\partial \mathbf{n}_{2}} & \text { on } \Gamma^{\text {in }} \\
\nu \frac{\partial u_{2}^{\lambda}}{\partial \mathbf{n}_{2}}-\mathbf{b} \cdot \mathbf{n}_{2} u_{2}^{\lambda} & \text { on } \Gamma^{\text {out }},\end{cases}\right.
$$

(actually $\mathcal{S}_{1}^{0} \lambda$ depends only on $\lambda^{i n}$ ) and $\mathcal{S}^{0}=\mathcal{S}_{1}^{0}+\mathcal{S}_{2}^{0}$ on $\Lambda_{2}$, such that

$$
\left\langle\left\langle\mathcal{S}^{0} \lambda, \mu\right\rangle\right\rangle_{\Gamma}=\left\langle\left\langle\mathcal{S}_{1}^{0} \lambda, \mu\right\rangle\right\rangle_{\Gamma}+\left\langle\left\langle\mathcal{S}_{2}^{0} \lambda, \mu\right\rangle\right\rangle_{\Gamma}
$$

Since $u_{1}^{\lambda}=u_{2}^{\lambda}=\lambda$ on $\Gamma^{i n}$, the Steklov-Poincaré equation (4.6) can also be written as

$$
\text { find } \lambda \in \Lambda_{2}: \quad\left\langle\left\langle\mathcal{S}^{0} \lambda, \mu\right\rangle\right\rangle_{\Gamma}=\langle\langle\chi, \mu\rangle\rangle_{\Gamma} \quad \forall \mu \in \Lambda_{2} .
$$

Remark 4.1. In view of Theorem 2.3, it is straightforward to prove that the operators $\mathcal{S}_{2}^{0}, \mathcal{S}$ and $\mathcal{S}^{0}$ are coercive on $H_{00}^{1 / 2}(\Gamma)$, whereas $\mathcal{S}_{2}$ is coercive only if smallness assumption for $\mathbf{b}$ are assumed, as required in (2.24). (See [18, 22] for a proof.)

Remark 4.2. The Steklov-Poincaré equations (4.6) and (4.11) realize the interface conditions IC1 (2.9) and IC2 (2.11), respectively, and they are equivalent one another at continuous level. Nevertheless they might not coincide at discrete level, when nonconforming discretization across the interface $\Gamma$ is used (see [5]). Moreover, their discrete counterparts feature a different computational performance, in particular for what concerns preconditioner efficiency. 


\subsection{The interface problem associated with XVF}

Let us start by writing the saddle-point problem (3.9) in terms of $u_{1}, u_{2}$ and $t$.

It reads: seek $u_{1} \in V_{1}^{0}, u_{2} \in V_{2}^{0}, t \in \Lambda_{1}^{\prime}$ such that

$$
\begin{cases}a_{1}\left(u_{1}, v_{1}\right)+{ }_{\Lambda_{1}^{\prime}}\left\langle t, v_{1}\right\rangle_{\Lambda_{1}}=\int_{\Omega_{1}} f v_{1} d \Omega & \forall v_{1} \in V_{1}^{0} \\ a_{2}\left(u_{2}, v_{2}\right)+d_{1}\left(u_{1}, v_{2}\right)-{ }_{\Lambda_{1}^{\prime}}\left\langle t, v_{2}\right\rangle_{\Lambda_{1}}=\int_{\Omega_{2}} f v_{2} d \Omega & \forall v_{2} \in V_{2}^{0} \\ \Lambda_{1}^{\prime}\left\langle s, u_{1}-u_{2}\right\rangle_{\Lambda_{1}}=0 & \forall s \in \Lambda_{1}^{\prime},\end{cases}
$$

where $a_{1}: V_{1}^{0} \times V_{1}^{0} \rightarrow \mathbb{R}:$

$$
a_{1}\left(u_{1}, v_{1}\right)=\int_{\Omega_{1}}\left[\operatorname{div}\left(\mathbf{b} u_{1}\right)+b_{0} u_{1}\right] v_{1} d \Omega-\alpha \int_{\Gamma^{i n}} \mathbf{b} \cdot \mathbf{n}_{\Gamma} u_{1} v_{1} d \Gamma
$$

$a_{2}: V_{2}^{0} \times V_{2}^{0} \rightarrow \mathbb{R}:$

$a_{2}\left(u_{2}, v_{2}\right)=\int_{\Omega_{2}} \nu \nabla u_{2} \cdot \nabla v_{2} d \Omega-\int_{\Omega_{2}} u_{2} \mathbf{b} \cdot \nabla v_{2} d \Omega+\int_{\Omega_{2}} b_{0} u_{2} v_{2} d \Omega-(1-\alpha) \int_{\Gamma^{i n}} \mathbf{b} \cdot \mathbf{n}_{\Gamma} u_{2} v_{2} d \Gamma$,

with $\alpha=0,1$ and $d_{1}: V_{1}^{0} \times V_{2}^{0} \rightarrow \mathbb{R}:$

$$
d_{1}\left(u_{1}, v_{2}\right)=-\int_{\Gamma^{\text {out }}} \mathbf{b} \cdot \mathbf{n}_{1} u_{1} v_{2} d \Gamma
$$

By decomposing both $u_{1}$ and $u_{2}$ as done in (4.1), problem (4.12) reads: seek $\lambda_{1} \in \Lambda_{1}, \lambda_{2} \in \Lambda_{2}, t \in \Lambda_{1}^{\prime}$ such that

$$
\left\{\begin{array}{cc}
a_{1}\left(u_{1}^{\lambda_{1}}, v_{1}^{\mu_{1}}\right)+\Lambda_{1}^{\prime}\left\langle t, v_{1}^{\mu_{1}}\right\rangle_{\Lambda_{1}}=\int_{\Omega_{1}} f v_{1}^{\mu_{1}} d \Omega-a_{1}\left(u_{1}^{f}, v_{1}^{\mu_{1}}\right) & \forall \mu_{1} \in \Lambda_{1} \\
a_{2}\left(u_{2}^{\lambda_{2}}, v_{2}^{\mu_{2}}\right)+d_{1}\left(u_{1}^{\lambda_{1}}, v_{2}^{\mu_{2}}\right)-{ }_{\Lambda_{1}^{\prime}}\left\langle t, v_{2}^{\mu_{2}}\right\rangle_{\Lambda_{1}} & \\
=\int_{\Omega_{2}} f v_{2}^{\mu_{2}} d \Omega-a_{2}\left(u_{2}^{f}, v_{2}^{\mu_{2}}\right)-d_{1}\left(u_{1}^{f}, v_{2}^{\mu_{2}}\right) & \forall \mu_{2} \in \Lambda_{2} \\
\Lambda_{1}^{\prime}\left\langle s, u_{1}^{\lambda_{1}}-u_{2}^{\lambda_{2}}\right\rangle_{\Lambda_{1}}=0 & \forall s \in \Lambda_{1}^{\prime} .
\end{array}\right.
$$

We consider the Steklov-Poincaré operators previously introduced.

Moreover, we denote by $\mathcal{S}_{1}^{\text {in }}$ and $\mathcal{S}_{1}^{\text {out }}$ the restrictions of the operator $\mathcal{S}_{1}$ to $\Gamma^{\text {in }}$ and $\Gamma^{\text {out }}$, respectively. Therefore, we note that

$$
\begin{array}{ll}
a_{1}\left(u_{1}^{\lambda_{1}}, v_{1}^{\mu_{1}}\right)=\alpha \int_{\Gamma^{\text {in }}}-\mathbf{b} \cdot \mathbf{n}_{1} \lambda_{1} \mu_{1} d \Gamma=\alpha\left\langle\mathcal{S}_{1}^{\text {in }} \lambda_{1}, \mu_{1}\right\rangle_{\Gamma^{\text {in }}} & \forall \mu_{1} \in \Lambda_{1}, \\
d_{1}\left(u_{1}^{\lambda_{1}}, v_{2}^{\mu_{2}}\right)=\int_{\Gamma^{\text {out }}}-\mathbf{b} \cdot \mathbf{n}_{1} u_{1}^{\lambda_{1}} \mu_{2} d \Gamma=\left\langle\left\langle\mathcal{S}_{1}^{\text {out }} \lambda_{1}, \mu_{2}\right\rangle\right\rangle_{\Gamma^{\text {out }}} & \forall \mu_{2} \in \Lambda_{2},
\end{array}
$$

where $\langle\cdot, \cdot\rangle_{\Gamma^{\text {in }}}$ is an abridged notation for $\Lambda_{1}^{\prime}\langle\cdot, \cdot\rangle_{\Lambda_{1}}$, while

$$
a_{2}\left(u_{2}^{\lambda_{2}}, v_{2}^{\mu_{2}}\right)=\alpha\left\langle\left\langle\mathcal{S}_{2} \lambda_{2}, \mu_{2}\right\rangle\right\rangle_{\Gamma}+(1-\alpha)\left\langle\left\langle\mathcal{S}_{2}^{0} \lambda_{2}, \mu_{2}\right\rangle\right\rangle_{\Gamma}
$$

and we set $\mathcal{S}_{2}^{\alpha}=\alpha \mathcal{S}_{2}+(1-\alpha) \mathcal{S}_{2}^{0}$, for $\alpha=0,1$. 
The interface problem equivalent to (4.13) takes the following form: seek $\lambda_{1} \in \Lambda_{1}, \lambda_{2} \in \Lambda_{2}, t \in \Lambda_{1}^{\prime}$ such that

$$
\begin{cases}\alpha\left\langle\mathcal{S}_{1}^{\text {in }} \lambda_{1}, \mu_{1}\right\rangle_{\Gamma^{\text {in }}}+\left\langle t, \mu_{1}\right\rangle_{\Gamma^{\text {in }}}=0 & \forall \mu_{1} \in \Lambda_{1} \\ \left\langle\left\langle\mathcal{S}_{2}^{\alpha} \lambda_{2}, \mu_{2}\right\rangle\right\rangle_{\Gamma}+\left\langle\left\langle\mathcal{S}_{1}^{\text {out }} \lambda_{1}, \mu_{2}\right\rangle\right\rangle_{\Gamma^{\text {out }}}-\left\langle t, \mu_{2}\right\rangle_{\Gamma^{\text {in }}}=\left\langle\left\langle\chi_{2}, \mu_{2}\right\rangle\right\rangle_{\Gamma}+\left\langle\left\langle\chi_{1}, \mu_{2}\right\rangle\right\rangle_{\Gamma^{\text {out }}} & \forall \mu_{2} \in \Lambda_{2}(4.14) \\ \left\langle s, \lambda_{1}-\lambda_{2}\right\rangle_{\Gamma^{\text {in }}}=0 & \forall s \in \Lambda_{1}^{\prime},\end{cases}
$$

where $\chi_{1}$ and $\chi_{2}$ are the fluxes on $\Gamma$ associated to $u_{1}^{f}$ and $u_{2}^{f}$, respectively, already defined in (4.8).

For brevity, we name (4.14) extended interface problem (XIP).

We can formally write system (4.14) in terms of a block-matrix operator as

$$
\text { find } \boldsymbol{\lambda} \in \Lambda_{1} \times \Lambda_{1}^{\prime} \times \Lambda_{2} \quad\left\langle\mathcal{A}_{D D}^{\alpha} \boldsymbol{\lambda}, \boldsymbol{\mu}\right\rangle=\langle\boldsymbol{\chi}, \boldsymbol{\mu}\rangle \quad \forall \boldsymbol{\mu} \in \Lambda_{1} \times \Lambda_{1}^{\prime} \times \Lambda_{2}
$$

with

$\mathcal{A}_{D D}^{\alpha}=$\begin{tabular}{|r|r|r|c|}
\hline$\alpha \mathcal{S}_{1}^{\text {in }}$ & $I$ & 0 & 0 \\
\hdashline$I$ & 0 & $-I$ & 0 \\
\hline 0 & $-I$ & & \\
\hdashline $\mathcal{S}_{1}^{\text {out }}$ & 0 & & \multicolumn{2}{|c|}{} \\
& & & \\
\hline
\end{tabular}
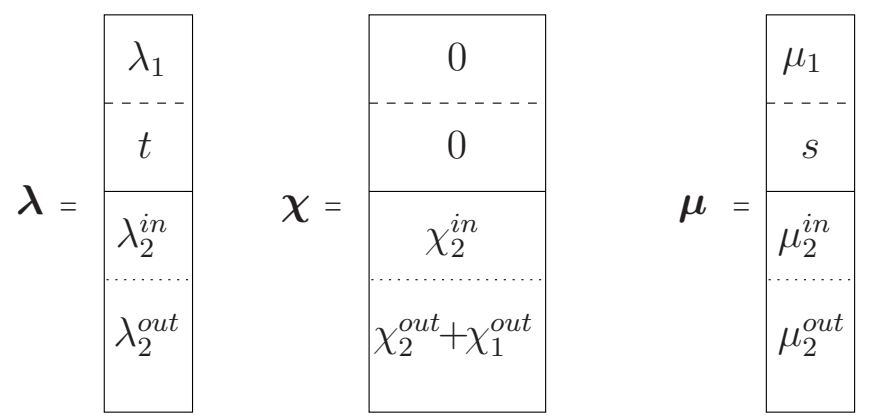

and where the upper-script in (out, resp.) denotes the restriction of the function to $\Gamma^{\text {in }}$ ( $\Gamma^{\text {out }}$, resp.).

Remark 4.3. Problem (4.15) is well-posed and $\boldsymbol{\lambda}$ is indeed the trace of the unique solution of (3.9).

The lower-script $D D$ stands for Dirichlet-Dirichlet. This notation is motivated by noting that, if $\lambda_{1}$ and $\lambda_{2}$ are known trace functions on $\Gamma^{i n}$ and $\Gamma$, respectively, then the evaluation of the matrix-vector product $\mathcal{A}_{D D}^{\alpha} \boldsymbol{\lambda}$ inside Bi-CGStab iterations requires the approximate solution of two Dirichlet problems like (4.3) (in $\Omega_{1}$ and $\Omega_{2}$, respectively) and the numerical evaluation of the fluxes across the interface $\Gamma$.

In view of the numerical discretization of problem (3.9) via interface problems like (4.15), a natural question arising from the analysis of the block-matrix system (4.15) concerns the possibility of replacing the operator $\mathcal{A}_{D D}^{\alpha}$ with another one involving Neumann interface conditions instead of Dirichlet ones.

To this aim, thanks to Theorems 2.2 and 2.3, we observe that both $\mathcal{S}_{1}^{i n}$ is invertible, and, if the smallness assumption (2.24) on $\mathbf{b}$ is satisfied, $\mathcal{S}_{2}$ is invertible too. Under assumption (2.24), by defining the (unknown) fluxes

$$
\left(H_{00}^{1 / 2}\left(\Gamma^{i n}\right)\right)^{\prime} \ni \phi_{1}=S_{1}^{i n} \lambda_{1}, \quad\left(H_{00}^{1 / 2}(\Gamma)\right)^{\prime} \ni \phi_{2}=S_{2}^{\alpha} \lambda_{2},
$$

we can split the unknown functions $u_{1}$ and $u_{2}$ as

$$
u_{1}=\tilde{u}_{1}^{\phi_{1}}+u_{1}^{f}, \quad u_{2}=\tilde{u}_{2}^{\phi_{2}}+u_{2}^{f},
$$


where $u_{1}^{f}$ and $u_{2}^{f}$ still denote the solutions of problems (4.2), while $\tilde{u}_{1}^{\phi_{1}}$ and $\tilde{u}_{2}^{\phi_{2}}$ are the solutions of the Neumann problems

$$
\left\{\begin{array} { l l } 
{ A _ { 1 } \tilde { u } _ { 1 } ^ { \phi _ { 1 } } = 0 } & { \text { in } \Omega _ { 1 } } \\
{ \tilde { u } _ { 1 } ^ { \phi _ { 1 } } = 0 } & { \text { on } ( \partial \Omega _ { 1 } \backslash \Gamma ) ^ { i n } } \\
{ - \mathbf { b } \cdot \mathbf { n } \tilde { u } _ { 1 } ^ { \phi _ { 1 } } = \phi _ { 1 } } & { \text { on } \Gamma ^ { i n } , }
\end{array} \quad \left\{\begin{array}{ll}
A_{2} \tilde{u}_{2}^{\phi_{2}}=0 & \text { in } \Omega_{2} \\
\tilde{u}_{2}^{\phi_{2}}=0 & \text { on } \partial \Omega_{2} \backslash \Gamma \\
\nu \frac{\partial \tilde{u}_{2}^{\phi_{2}}}{\partial \mathbf{n}_{2}}-\mathbf{b} \cdot \mathbf{n}_{2} \tilde{u}_{2}^{\phi_{2}}=\phi_{2} & \text { on } \Gamma^{\text {out }} \\
\nu \frac{\partial \tilde{u}_{2}^{\phi_{2}}}{\partial \mathbf{n}_{2}}-\alpha \mathbf{b} \cdot \mathbf{n}_{2} \tilde{u}_{2}^{\phi_{2}}=\phi_{2} & \text { on } \Gamma^{\text {in }}
\end{array}\right.\right.
$$

Problem (4.12) can be reformulated in terms of the (unknown) flux variables $\phi_{1}$ and $\phi_{2}$.

$$
\text { find } \boldsymbol{\phi} \in \Lambda_{1}^{\prime} \times \Lambda_{1}^{\prime} \times \Lambda_{2}^{\prime} \quad\left\langle\mathcal{A}_{N N}^{\alpha} \boldsymbol{\phi}, \boldsymbol{\mu}\right\rangle=\langle\chi, \boldsymbol{\mu}\rangle \quad \forall \boldsymbol{\mu} \in \Lambda_{1} \times \Lambda_{1}^{\prime} \times \Lambda_{2}
$$

with

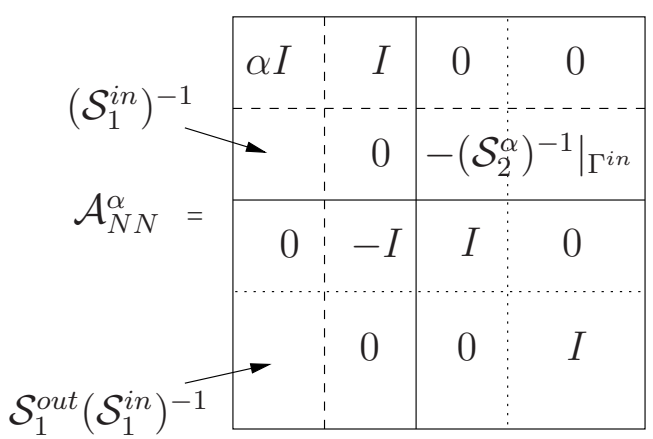

$$
\phi=\mid \begin{array}{c|}
\hline \phi_{1} \\
-t \\
\hline \phi_{2}^{\text {in }} \\
\cdots \cdots \\
\phi_{2}^{\text {out }} \\
\hline
\end{array}
$$

while $\boldsymbol{\mu}$ and $\boldsymbol{\chi}$ have the same meaning as above.

In this case, the evaluation of the matrix vector product $\mathcal{A}_{N N}^{\alpha} \phi$ requires the solution of two Neumann problems (like (4.18)) and then the evaluation of the trace of $\tilde{u}_{1}^{\phi_{1}}\left(\tilde{u}_{2}^{\phi_{2}}\right.$, resp.) on $\Gamma^{i n}\left(\Gamma\right.$, resp.), together with the evaluation of the flux $-\mathbf{b} \cdot \mathbf{n} \tilde{u}_{1}^{\phi_{1}}$ on $\Gamma^{o u t}$. For this reason, the formulation (4.19) is named Neumann-Neumann.

Remark 4.4. When the smallness assumption (2.24) is not satisfied, the elliptic problem in (4.18) is not always well-posed and instabilities can develop (see Fig. 5.4). The same drawback characterizes the next Dirichlet-Neumann form, too.

The survey can be completed by considering either Dirichlet-Neumann or NeumannDirichlet formulations for problem (4.12), depending on the choice of either Dirichlet or Neumann unknowns at the interface.

More precisely, we name Dirichlet-Neumann (Neumann-Dirichlet, resp.) the formulation in which we decompose the unknown functions $u_{1}$ and $u_{2}$ as $u_{1}=u_{1}^{\lambda_{1}}+u_{1}^{f}, u_{2}=\tilde{u}_{2}^{\phi_{2}}+u_{2}^{f}$ $\left(u_{1}=\tilde{u}_{1}^{\phi_{1}}+u_{1}^{f}, u_{2}=u_{2}^{\lambda_{2}}+u_{2}^{f}\right.$, resp.). The corresponding systems at the interface read:

find $\boldsymbol{\psi}_{D N} \in \Lambda_{1} \times \Lambda_{1}^{\prime} \times \Lambda_{2}^{\prime} \quad\left\langle\mathcal{A}_{D N}^{\alpha} \boldsymbol{\psi}_{D N}, \boldsymbol{\mu}\right\rangle=\langle\chi, \boldsymbol{\mu}\rangle \quad \forall \boldsymbol{\mu} \in \Lambda_{1} \times \Lambda_{1}^{\prime} \times \Lambda_{2}$

and

find $\boldsymbol{\psi}_{N D} \in \Lambda_{1}^{\prime} \times \Lambda_{1}^{\prime} \times \Lambda_{2} \quad\left\langle\mathcal{A}_{N D}^{\alpha} \psi_{N D}, \boldsymbol{\mu}\right\rangle=\langle\chi, \boldsymbol{\mu}\rangle \quad \forall \boldsymbol{\mu} \in \Lambda_{1} \times \Lambda_{1}^{\prime} \times \Lambda_{2}$

with $\boldsymbol{\psi}_{D N}=\left[\lambda_{1}, t,\left(\phi_{2}^{\text {in }}, \phi_{2}^{\text {out }}\right)\right]^{t}, \boldsymbol{\psi}_{N D}=\left[\phi_{1}, t,\left(\lambda_{2}^{\text {in }}, \lambda_{2}^{\text {out }}\right)\right]^{t}$ and 


$\mathcal{A}_{D N}^{\alpha}=$\begin{tabular}{|r|c|c|c|}
\hline $\mathcal{S}_{1}^{\text {in }}$ & $I$ & 0 & 0 \\
\hdashline 0 & $I$ & $-\left.\left(\mathcal{S}_{2}^{\alpha}\right)^{-1}\right|_{\Gamma^{\text {in }}}$ \\
\hline 0 & $-I$ & $I$ & 0 \\
\hdashline $\mathcal{S}_{1}^{\text {out }}$ & 0 & 0 & $I$ \\
\hline
\end{tabular}

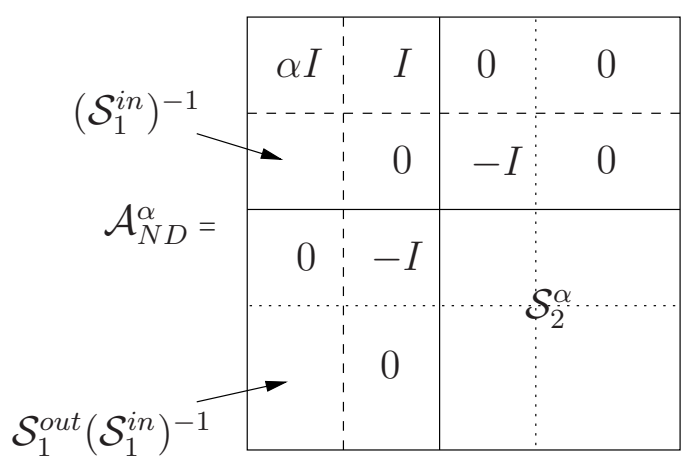

As for the NN form, DN form is well-posed if the smallness assumption (2.24) on $\mathbf{b}$ is assumed. It is worth repeating that (4.15), (4.19), (4.20) and (4.21) represent alternative (albeit equivalent) interface formulations for the extended problem (3.9). Their finite dimensional approximations will however yield different numerical solutions, and the algebraic counterparts will give rise to systems whose computational solution features different levels of complexity, see Section 5.3.

\section{The discretized problems}

In this section we describe the discretization of the variational problems discussed in the previous sections and we propose optimal preconditioners to efficiently solve the associated linear systems.

\subsection{Spectral Element discretization}

The discretization of the differential equation within each subdomain is performed by quadrilateral conformal Spectral Element Methods (SEM). We refer to [9] for a detailed description of these methods, here we briefly recall their basic features.

For $k=1,2$, let $\mathcal{T}_{k}=\left\{T_{k, m}\right\}_{m=1}^{M_{k}}$ be a partition of the computational domain $\Omega_{k} \subset \mathbb{R}^{d}(d=$ $2,3)$, where each element $T_{k, m}$ is obtained by a bijective and differentiable transformation $\mathbf{F}_{k, m}$ from the reference (or parent) element $\hat{\Omega}^{d}=(-1,1)^{d}$. We suppose that two adjacent elements of $\mathcal{T}_{k}$ share either a common vertex or a complete side. On the reference element we define the finite dimensional space $\hat{\mathbb{Q}}_{N}=\operatorname{span}\left\{\hat{x}_{1}^{j_{1}} \cdots \hat{x}_{d}^{j_{d}}: 0 \leqslant j_{1}, \ldots, j_{d} \leqslant N\right\}$ and, for any $T_{k, m} \in \mathcal{T}_{k}: \quad T_{k, m}=\mathbf{F}_{k, m}\left(\hat{\Omega}^{d}\right)$, set $h_{k, m}=\operatorname{diam}\left(T_{k, m}\right)$ and

$$
V_{N_{k}}\left(T_{k, m}\right)=\left\{v: v=\hat{v} \circ \mathbf{F}_{k, m}^{-1} \text { for some } \hat{v} \in \hat{\mathbb{Q}}_{N_{k}}\right\}
$$

The SEM multidimensional space on $\bar{\Omega}_{k}($ for $k=1,2)$ is

$$
X_{k, \delta_{k}}=\left\{v \in C^{0}\left(\bar{\Omega}_{k}\right): v_{\mid T_{k, m}} \in V_{N_{k}}\left(T_{k, m}\right), \forall T_{k, m} \in \mathcal{T}_{k}\right\}
$$

where $\delta_{k}$ is an abridged notation for "discrete", that accounts for the local geometric sizes $\left\{h_{k, m}\right\}$ and the local polynomial degrees $\left\{N_{k}\right\}$, for $m=1, \ldots, M_{k}$ and $k=1,2$. Note that the polynomial degree $N_{k}$ can either coincide or differ along each spatial direction. In the latter case we denote by $N_{k}^{x}, N_{k}^{y}$ and $N_{k}^{z}$ the polynomial degrees along $x, y$ and $z$ directions, respectively. $N_{k}^{x}, N_{k}^{y}$ and $N_{k}^{z}$ can change from one spectral element to another, however both geometric and approximation conformity is guaranteed inside $\bar{\Omega}_{k}$. 
The finite dimensional spaces in which we look for the Spectral Element solution are defined as follows (see (3.1), (3.2) and (3.3) for definitions of $V_{k}, V_{k}^{0}, \Lambda_{k}$ ):

$$
\begin{aligned}
& V_{k, \delta_{k}}=V_{k} \cap X_{k, \delta_{k}}, \quad V_{k, \delta_{k}}^{0}=V_{k}^{0} \cap X_{k, \delta_{k}}, \quad k=1,2 \\
& \Lambda_{1, \delta_{1}}=\left\{\left.v_{1, \delta_{1}}\right|_{\Gamma^{i n}}: v_{1, \delta_{1}} \in V_{1, \delta_{1}}\right\}, \quad \Lambda_{2, \delta_{2}}=\left\{\left.v_{2, \delta_{2}}\right|_{\Gamma}: v_{2, \delta_{2}} \in V_{2, \delta_{2}}\right\} .
\end{aligned}
$$

Because of the difficulty to compute integrals exactly, the bilinear forms $a_{1}, a_{2}, d_{1}$, the duality products between $\Lambda_{k}$ and $\Lambda_{k}^{\prime}$ (for $k=1,2$ ), and the $L^{2}$-inner products are all approximated by Legendre-Gauss-Lobatto (LGL) formulas on the grid induced by the finite dimensional spaces defined above. It is well known (see [8]) that the quadrature error introduced behaves like the approximation error induced by the spectral approximation. The abridged notation SEM-NI stands for Spectral Element Method with Numerical Integration.

Coherently, we can define the finite dimensional counterpart of the Steklov-Poincaré operators $\mathcal{S}_{1}, \mathcal{S}_{2}$, etc.. To this aim, for $k=1,2$, we denote by $\mathcal{N}_{k, \Gamma}$ the set of nodes of $\mathcal{T}_{k} \cap \Gamma$ whose cardinality is $N_{k, \Gamma}$. Similar notations are used for the nodes lying on either $\Gamma^{i n}$ or $\Gamma^{\text {out }}$.

The finite dimensional basis in $\Lambda_{1, \delta_{1}}$ is generated by the characteristic Lagrange polynomials in $\Omega_{1}$ associated to the LGL nodes of $\mathcal{N}_{1, \Gamma^{i n}}$, while that in $\Lambda_{2, \delta_{2}}$ is generated by the characteristic Lagrange polynomials in $\Omega_{2}$ associated to the LGL nodes of $\mathcal{N}_{2, \Gamma}$. We denote by $\mu_{1}^{(i)}, i=1, \ldots, N_{1, \Gamma^{i n}}$ the basis functions of $\Lambda_{1, \delta_{1}}$ and by $\mu_{2}^{(i)}, i=1, \ldots, N_{2, \Gamma}$ the basis functions of $\Lambda_{2, \delta_{2}}$.

To span the dual spaces $\Lambda_{k, \delta_{k}}^{\prime}$ we use the same Lagrange basis of $\Lambda_{k, \delta_{k}}$, respectively for $k=1,2$. It is immediate to prove that, under regularity assumptions (2.3) and (2.16), $\Lambda_{k, \delta_{k}}^{\prime} \subset \Lambda_{k}^{\prime}$, for $k=1,2$.

Then we set

$$
\begin{array}{lll}
\left(S_{1, \delta_{1}}^{\text {in }}\right)_{i j} & =\left\langle\mathcal{S}_{1}^{\text {in }} \mu_{1}^{(j)}, \mu_{1}^{(i)}\right\rangle_{\Gamma^{\text {in }}} & i, j=0, \ldots, N_{1, \Gamma^{\text {in }}} \\
\left(S_{1, \delta_{1}}^{\text {out }}\right)_{i j}=\left\langle\mathcal{S}_{1}^{\text {out }} \mu_{1}^{(j)}, \mu_{2}^{(i)}\right\rangle_{\Gamma^{\text {out }}} & i=0, \ldots, N_{2, \Gamma^{\text {out }}}, j=0, \ldots, N_{1, \Gamma^{\text {in }}} \\
\left(S_{2, \delta_{2}}\right)_{i j}=\left\langle\mathcal{S}_{2} \mu_{2}^{(j)}, \mu_{2}^{(i)}\right\rangle_{\Gamma} & i, j=0, \ldots, N_{2, \Gamma} \\
\left(S_{2, \delta_{2}}^{0}\right)_{i j}=\left\langle\mathcal{S}_{2}^{0} \mu_{2}^{(j)}, \mu_{2}^{(i)}\right\rangle_{\Gamma} & i, j=0, \ldots, N_{2, \Gamma} .
\end{array}
$$

In general, the subscript $\delta$ denotes the finite dimensional counterpart of the corresponding operator defined at the continuous level and, even if it is not specified, we understand that it is constructed following the SEM-NI approximation. For an extensive presentation of these methods we refer to $[8,9]$.

Remark 5.1. If the two partitions $\mathcal{T}_{1}$ and $\mathcal{T}_{2}$ share the same edges on $\Gamma$ and the polynomial degrees coincide in the hyperbolic domain $\Omega_{1}$ and in the elliptic one $\Omega_{2}$, we call that conforming discretization or conforming coupling across $\Gamma$. In this case $N=N_{1, \Gamma}=N_{2, \Gamma}$ denotes the common polynomial degree and $\delta=\delta_{1}=\delta_{2}$ denotes the conforming discretization.

Otherwise, we call nonconforming discretization or nonconforming coupling across $\Gamma$ the case in which either the partitions $\mathcal{T}_{1}$ and $\mathcal{T}_{2}$ do not share the same edges on $\Gamma$ or the polynomial degrees $N_{1, \Gamma}$ and $N_{2, \Gamma}$ differ each other. In this work we bound our analysis to conforming couplings, while we refer to [5] for the nonconforming case, where among all the known methods dealing with nonconformity we choose mortar methods (see, e.g., $[2,1]$ ), since they achieve the constraint equation associated to the XVF in a very natural way.

The use of conforming discretization across the interface $\Gamma$ implies that the approximation of the unknown trace function is defined through a unique set of degrees of freedom on $\Gamma$ 
and the same symbol $\delta\left(=\delta_{1}=\delta_{2}\right)$ will be used. We denote by $M_{\delta}, M_{\delta}^{\text {in }}$ and $M_{\delta}^{\text {out }}$ the mass matrices associated to the interface integrals on $\Gamma, \Gamma^{\text {in }}$ and $\Gamma^{\text {out }}$, respectively. Due to the orthogonality of the characteristic Lagrange basis functions with respect to the GaussLegendre-Lobatto quadrature formulas, these mass matrices are diagonal.

The conforming finite dimensional counterpart of (4.6) reads:

$$
\text { find } \lambda_{\delta} \in \Lambda_{2, \delta}: \quad S_{\delta} \lambda_{\delta}=\chi_{\delta},
$$

while that of (4.11) reads:

$$
\text { find } \lambda_{\delta} \in \Lambda_{2, \delta}: \quad S_{\delta}^{0} \lambda_{\delta}=\chi_{\delta}
$$

After setting $\chi_{\delta}=\left[0,0, M_{\delta}^{\text {in }} \chi_{2, \delta}^{\text {in }}, M_{\delta}^{\text {out }}\left(\chi_{2, \delta}^{\text {out }}+\chi_{1, \delta}^{\text {out }}\right)\right]^{t}$, the discretized form corresponding to XIP problems (4.15), (4.19), (4.20) and (4.21) are formulated as follows:

\section{Dirichlet-Dirichlet}

$$
\text { find } \boldsymbol{\lambda}_{\delta}=\left[\lambda_{1, \delta}, t_{\delta},\left(\lambda_{2, \delta}^{i n}, \lambda_{2, \delta}^{\text {out }}\right)\right]^{t} \in \Lambda_{1, \delta} \times \Lambda_{1, \delta}^{\prime} \times \Lambda_{2, \delta}: \quad A_{D D, \delta}^{\alpha} \boldsymbol{\lambda}_{\delta}=\boldsymbol{\chi}_{\delta},
$$

Neumann-Neumann

$$
\text { find } \phi_{\delta}=\left[\phi_{1, \delta}, t_{\delta},\left(\phi_{2, \delta}^{i n}, \phi_{2, \delta}^{\text {out }}\right)\right]^{t} \in \Lambda_{1, \delta} \times \Lambda_{1, \delta}^{\prime} \times \Lambda_{2, \delta}: \quad A_{N N, \delta}^{\alpha} \phi_{\delta}=\chi_{\delta},
$$

Dirichlet-Neumann

$$
\text { find } \boldsymbol{\psi}_{D N, \delta}=\left[\lambda_{1, \delta}, t_{\delta},\left(\phi_{2, \delta}^{\text {in }}, \phi_{2, \delta}^{\text {out }}\right)\right]^{t} \in \Lambda_{1, \delta} \times \Lambda_{1, \delta}^{\prime} \times \Lambda_{2, \delta}: \quad A_{D D, \delta}^{\alpha} \boldsymbol{\psi}_{D N, \delta}=\boldsymbol{\chi}_{\delta}
$$

Neumann-Dirichlet

$$
\text { find } \boldsymbol{\psi}_{N D, \delta}=\left[\phi_{1, \delta}, t_{\delta},\left(\lambda_{2, \delta}^{\text {in }}, \lambda_{2, \delta}^{\text {out }}\right)\right]^{t} \in \Lambda_{1, \delta} \times \Lambda_{1, \delta}^{\prime} \times \Lambda_{2, \delta}: \quad A_{N D, \delta}^{\alpha} \boldsymbol{\psi}_{N D, \delta}=\boldsymbol{\chi}_{\delta}
$$

where
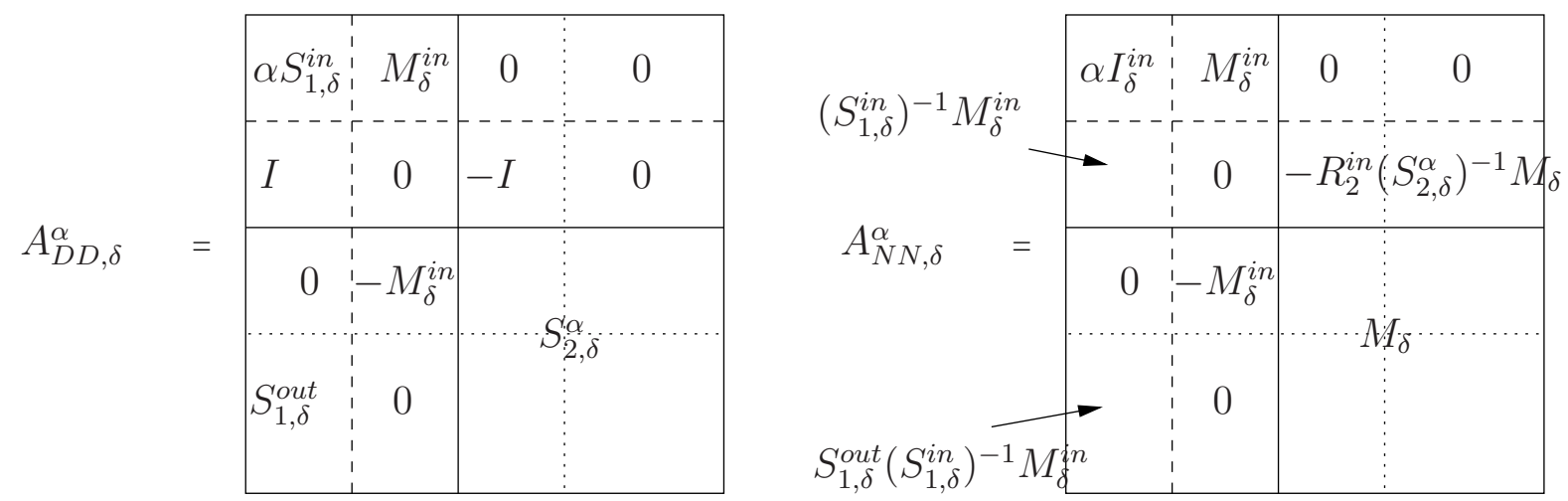
$R_{2}^{i n}$ is the restriction matrix from $\mathcal{N}_{2, \Gamma}$ to $\mathcal{N}_{2, \Gamma^{\text {in }}}$, and
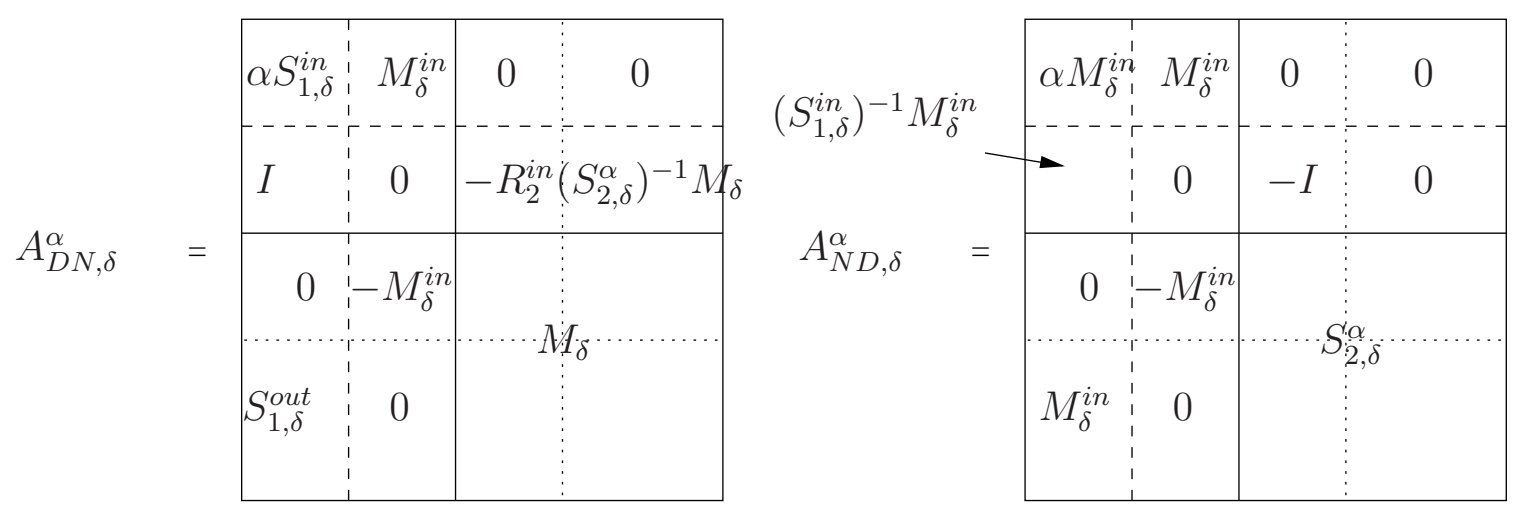

Remark 5.2. Let us consider the discrete counterpart of (3.9) seek $u_{\delta} \in V_{\delta}^{0}, t_{\delta} \in \Lambda_{1, \delta}^{\prime}$ :

$$
\begin{cases}a\left(u_{\delta}, v_{\delta}\right)+b\left(v_{\delta}, t_{\delta}\right)=F\left(v_{\delta}\right) & \forall v_{\delta} \in V_{\delta}^{0} \\ b\left(u_{\delta}, s_{\delta}\right)=0 & \forall s_{\delta} \in \Lambda_{1, \delta}^{\prime}\end{cases}
$$

where $V_{\delta}^{0}=V_{1, \delta}^{0} \times V_{2 \delta}^{0}$.

When the conforming discretization is considered across the interface, which is the case of the present work, the following inclusions hold: $V_{\delta}^{0} \subset V^{0}, \Lambda_{1, \delta_{1}}^{\prime} \subset \Lambda_{1}^{\prime}, Z_{\delta}=\left\{v_{\delta} \in V_{\delta}^{0}\right.$ : $v_{1, \delta}=v_{2, \delta}$ on $\left.\Gamma^{i n}\right\} \subset Z$, where $Z_{\delta}$ is the kernel of the discrete counterpart $B_{\delta}$ of $B$, that is $\Lambda_{1}^{\prime}\left\langle s_{\delta}, B_{\delta} v_{\delta}\right\rangle_{\Lambda_{1}}=b\left(v_{\delta}, s_{\delta}\right)$, for any $v_{\delta} \in V_{\delta}^{0}$ and $s_{\delta} \in \Lambda_{1, \delta}^{\prime}$.

The latter inclusion is crucial for the proof that the discrete operator $\pi A_{\delta}$ is an isomorphism from $Z_{\delta}$ onto $Z_{\delta}^{\prime}\left(\pi A_{\delta}\right.$ is defined by ${ }_{V^{\prime}}\left\langle\pi A_{\delta} u_{\delta}, v_{\delta}\right\rangle_{V}=a\left(u_{\delta}, v_{\delta}\right)$, for any $\left.u_{\delta}, v_{\delta} \in V_{\delta}^{0}\right)$ and it can be obtained by using the same arguments of $i$ ) in the proof of Theorem 3.1. Finally, in order that the discrete inf-sup condition corresponding to (3.10) holds, the surjectivity of $B_{\delta}$ can be proved as in step ii) of the proof of Theorem 3.1.

All the finite dimensional interface equations (5.4)-(5.7) can be solved by a Krylov method for non-symmetric systems (e.g., Bi-CGStab).

\subsection{Comparison among formulations. Numerical results}

In this section we compare the Steklov-Poincaré approaches SP0 (5.3) and SP (5.2) with the extended interface problems (XIP) (5.4), (5.5), (5.6) and (5.7) presented in this work in terms of Bi-CGStab iterations (see [24]) needed to solve the corresponding interface equations, versus the discretization parameters, both the polynomial degree $N$ and the number of spectral elements $M$ in each subdomain. More precisely, Steklov-Poincaré approach SP0 is related to the four XIP with $\alpha=0$, since all these forms provide interface conditions (2.11); similarly Steklov-Poincaré approach SP is related to XIP with $\alpha=1$, since all these forms provide interface conditions (2.9).

Let us consider the following test cases.

Test case \#1. The computational domain $\Omega=(-1,1)^{2}$ is split in $\Omega_{1}=(-1,0.8) \times(-1,1)$ and $\Omega_{2}=(0.8,1) \times(-1,1)$. The interface is $\Gamma=\{0.8\} \times(-1,1)$. The data of the problem are: $\mathbf{b}=[10 y, 0]^{t}, b_{0}=1, f=1$ and the inflow interface is $\Gamma^{i n}=\{0.8\} \times(-1,0)$. Dirichlet boundary conditions are imposed on the vertical sides of $\Omega$, precisely $u_{1}=1$ on $\left(\partial \Omega_{1} \backslash \Gamma\right)^{i n}=$ 

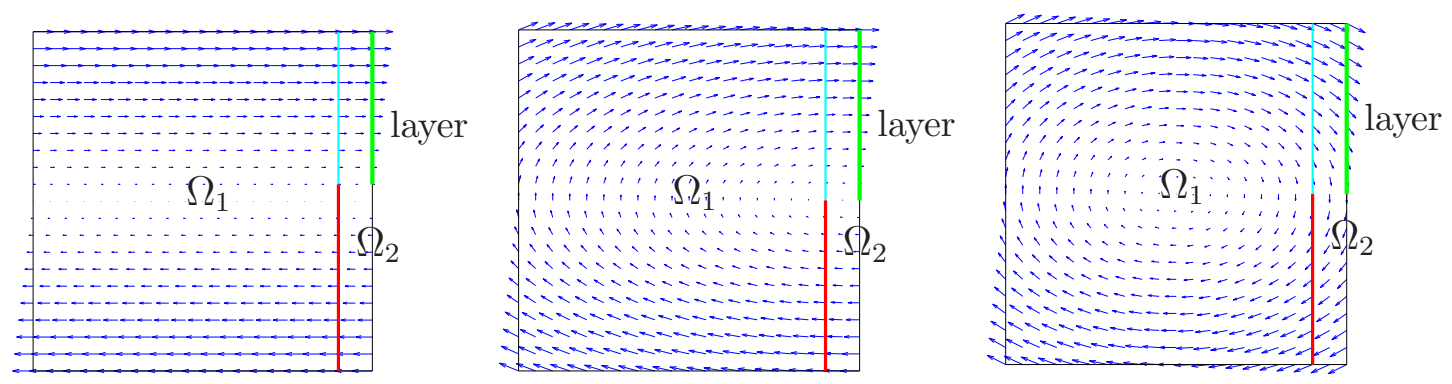

Figure 5.1. Data for test cases \#1 (left), \#2 (center) and \# 3 (right). The cyan (red, resp.) line denotes the interface $\Gamma^{\text {out }}\left(\Gamma^{\text {in }}\right.$, resp.)

$\{-1\} \times(0,1), u_{2}=0$ on $\{1\} \times(-1,1)$, while the homogeneous Neumann condition $\frac{\partial u_{2}}{\partial \mathbf{n}_{2}}=0$ is imposed on $((0.8,1) \times\{-1\}) \cup((0.8,1) \times\{1\})$ (see Fig. 5.1 left).

Test case \#2. The computational domain $\Omega=(-1,1)^{2}$ is split in $\Omega_{1}=(-1,0.8) \times(-1,1)$ and $\Omega_{2}=(0.8,1) \times(-1,1)$. The interface is $\Gamma=\{0.8\} \times(-1,1)$. The data of the problem are: $\mathbf{b}=[5 y, 1-x]^{t}, b_{0}=1, f=1$ and the inflow interface is $\Gamma^{i n}=\{0.8\} \times(-1,0)$. The imposed Dirichlet boundary conditions are: $u_{1}=1$ on $((-1,0.8) \times\{-1\}) \cup(\{-1\} \times(0,1)), u_{2}=0$ on $\{1\} \times(-1,1), u_{2}=1$ on $(0.8,1) \times\{-1\}$, while the homogeneous Neumann condition $\frac{\partial u_{2}}{\partial \mathbf{n}_{2}}=0$ is imposed on $(0.8,1) \times\{1\}$ (see Fig. 5.1 center).

Test case \#3. The computational domain $\Omega=(-1,1)^{2}$ is split in $\Omega_{1}=(-1,0.8) \times(-1,1)$ and $\Omega_{2}=(0.8,1) \times(-1,1)$. The interface is $\Gamma=\{0.8\} \times(-1,1)$. The data of the problem are: $\mathbf{b}=[2 y,-x]^{t}, b_{0}=1, f=1$ and the inflow interface is $\Gamma^{i n}=\{0.8\} \times(-1,0.1)$. The imposed Dirichlet boundary condition are: $u_{1}(x, y)=(1-x) / 2$ on $(-1,0) \times\{-1\}$, $u_{1}=1$ on $\{-1\} \times(0,1), u_{1}(x, y)=2 / \pi \arctan (1000(1-x))$ on $(0,0.8) \times\{1\}, u_{2}(x, y)=$ $2 / \pi \arctan (1000(1-x))$ on $(0.8,1) \times\{1\}, u_{2}=0$ on $\{1\} \times(-1,1)$. Finally the homogeneous Neumann condition $\frac{\partial u_{2}}{\partial \mathbf{n}_{2}}=0$ is imposed on $(0.8,1) \times\{-1\}$ (see Fig. 5.1 right).

For all the test cases, the viscosity will be specified below.

We discretize the problem using the conforming SEM-NI approach, presented in the previous Section. In each subdomain we define a mesh of quadrilaterals that can be either uniform or not. We remind that in this section and in the following one we consider conforming discretizations across the interface between the hyperbolic and the elliptic subdomains. Because of the presence of a boundary layer near the right vertical side, the mesh is finer there and a high polynomial degree along $x$ direction is used to prevent the numerical solution to be affected by spurious oscillations.

In Fig. 5.2 we compare the number of Bi-CGStab iterations for the Test case \#1 needed to solve the extended interface problems (5.4), (5.5), (5.6), (5.7) and the heterogeneous Steklov-Poincarè equations (5.2), (5.3) versus the spectral polynomial degree and the number of spectral elements, respectively. Denoting by $r^{(k)}$ the residual of the linear system at the $k$-th Bi-CGStab iteration, the iterations are stopped when $\left\|r^{(k+1)}\right\| /\left\|r^{(0)}\right\| \leqslant \epsilon$, with $\epsilon=10^{-12}$.

For each formulation, we have computed a least-square fit of a law like $\# i t \simeq C x^{q}$, where $x$ can indicate either the viscosity $\nu$, the spectral polynomial degree $N$ or the number of spectral elements $M$ along each direction and in each subdomain. In each legend we report the estimated value of $q$.

The convergence rate of the XIP approaches depends on the parameter $\alpha$, i.e., on the set of interface conditions chosen, (2.9) for $\alpha=1$ and (2.11) for $\alpha=0$. As a matter of 

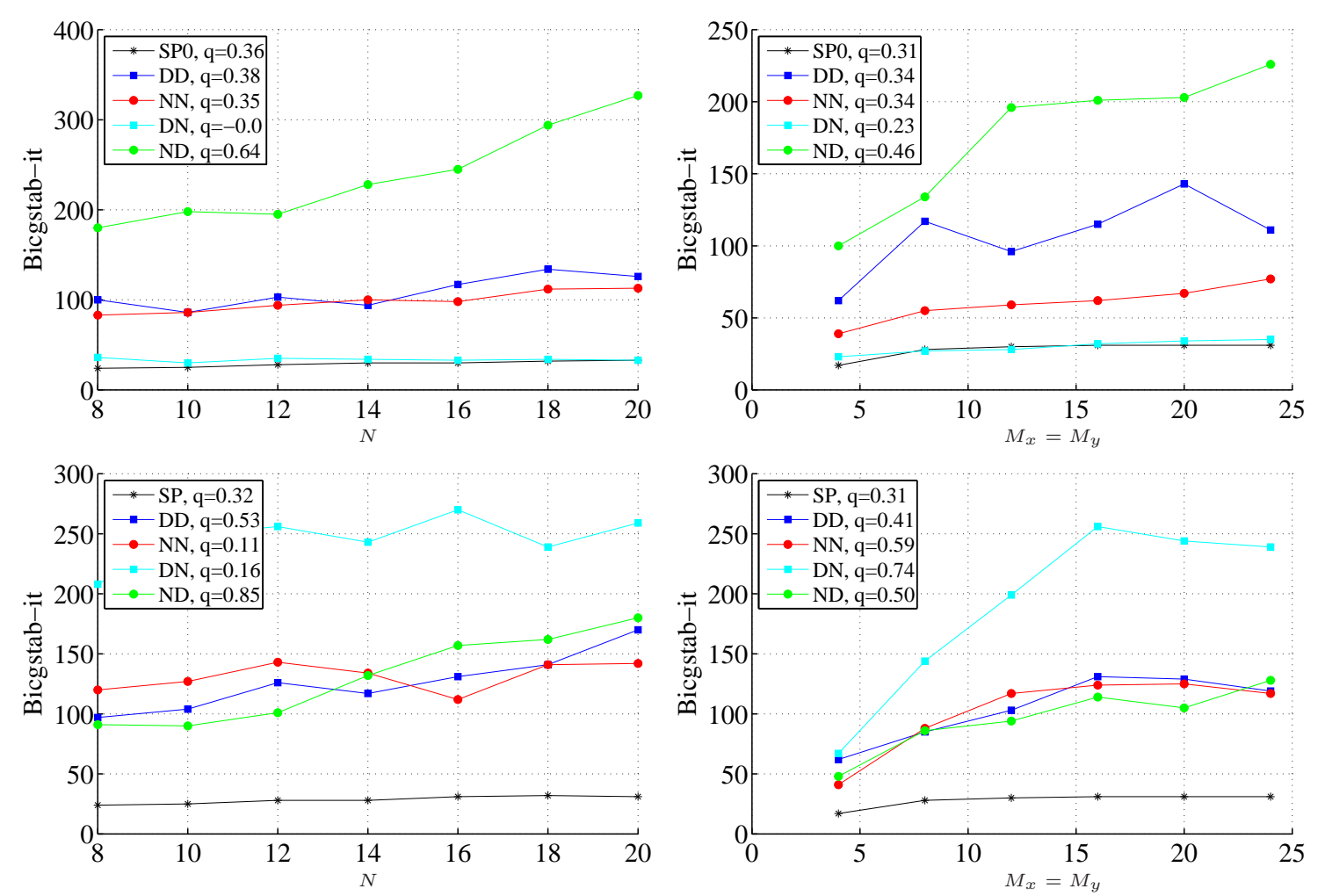

Figure 5.2. Test case \#1. Bi-CGStab iterations needed to solve the extended interface problems DD (5.4), NN (5.5), DN (5.6), ND (5.7) (at top with $\alpha=0$, at bottom with $\alpha=1$ ) and the Steklov Poincaré equations SP0 (5.3) and SP (5.2). The viscosity is $\nu=0.01$. At left, the spectral element mesh is formed by $3 \times 6$ (4 $\times 6$, resp.) elements in $\Omega_{1}\left(\Omega_{2}\right.$, resp.), while the polynomial degree is $N$ in each element of each domain. At right, the polynomial degree is $N=6$ in all elements, while the number of spectral elements in each $\Omega_{k}$ $(k=1,2)$ is $M=M_{x} \times M_{y}$

fact when $\alpha=0$, DD (as SP0) is the best approach, while ND is the worst one for what concerns the dependence on polynomial degree and number of spectral elements (see Fig. 5.2 , top). On the contrary, when $\alpha=1$, with the exception of DN form, all the other XIP forms outperform in a similar way, but the number of iterations are always lower for the BiCGStab solution applied to the classical (non-extended) interface problems (5.2) (cf. curve) (see Fig. 5.2, bottom).

In Fig. 5.3 we report the number of Bi-CGStab iterations versus the viscosity. It noticeably grows when $\nu \rightarrow 0$ and $\alpha=1$ (Fig. 5.3, right) for both NN and DN approaches. This is due to the fact that condition (2.24) is not satisfied when $\nu \lesssim 10^{-3}$ and instabilities affect the numerical solution, see also Remark 4.4. In Fig. 5.4 the numerical solution of Test \# 1 is shown for $\nu=10^{-4}$, unstable (stable, resp.) for NN (DD, resp.) form and $\alpha=1$. In some situations, the use of a good preconditioner will stabilize the solution, as we will see in the next section.

For what concerns the dependence on the viscosity, the convergence rate of all approaches is independent of $\nu$, with the exception of XIP approaches NN and DN when $\alpha=1$. Also in this case, the number of iterations are always lower for the Bi-CGStab solution applied to the classical (non-extended) interface problems (5.3) and (5.2) (cf. "SP0" and "SP" curves). 

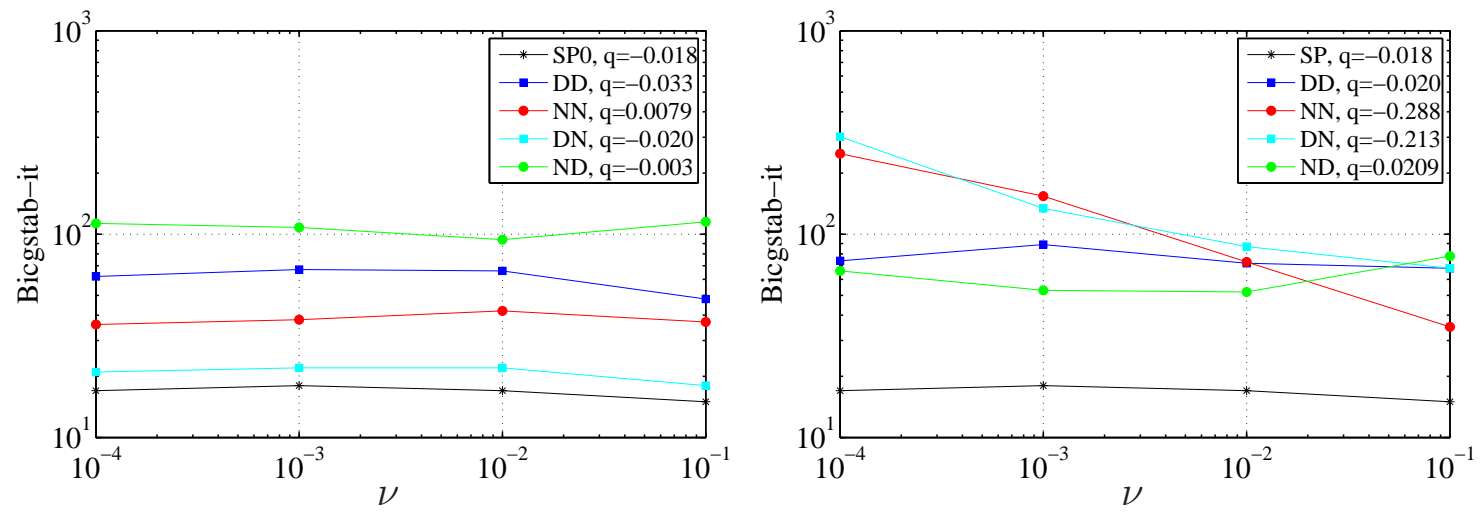

Figure 5.3. Test case \#1. Bi-CGStab iterations needed to solve the XIP DD (5.4), NN (5.5), DN (5.6), ND (5.7) (at left with $\alpha=0$, at right with $\alpha=1$ ) and the Steklov Poincaré equations SP0 (5.3) and SP (5.2). The spectral element mesh is generated by $3 \times 4$ elements with $N=6$ in $\Omega_{1}$, and by $6 \times 4$ elements in $\Omega_{2} . N=6$ in all elements of $\Omega_{2}$ with the exception of those close to the boundary layer (where $N_{2}^{x}=72$ $\left.N_{2}^{y}=6\right)$
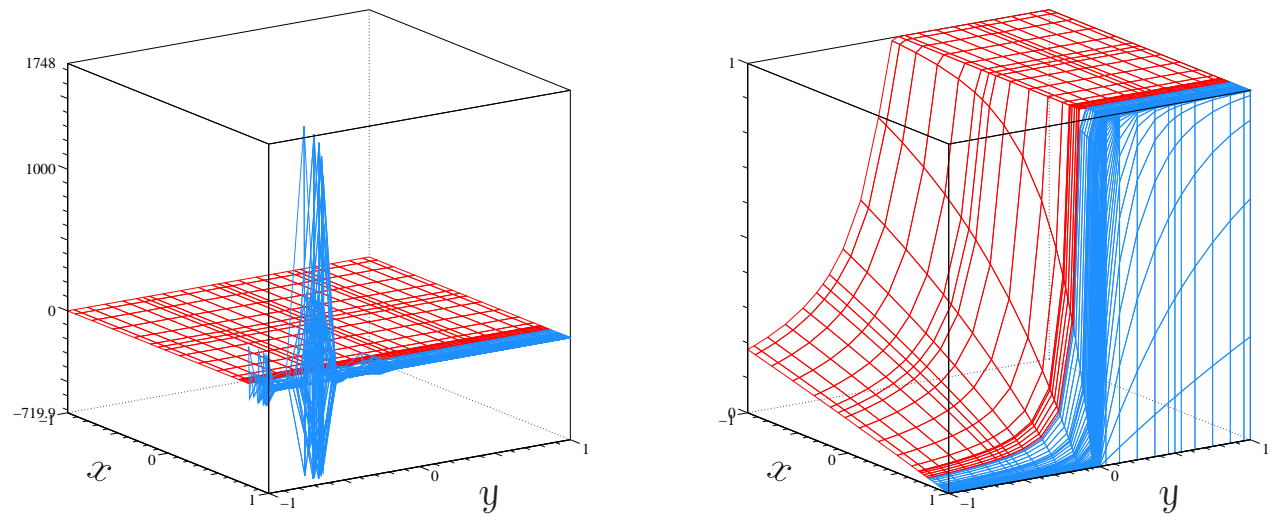

Figure 5.4. Test case \#1. Numerical solution provided by NN formulation (4.19) (at left) and by DD form (4.15) (at right) for $\nu=10^{-4}$ when $\alpha=1$

\subsection{Optimal preconditioners for the interface problems}

Starting from the well known results about preconditioning of the discrete Steklov-Poincaré operator $S_{\delta}^{0}(4.10)$, in this Section we propose optimal preconditioners for the finite dimensional counterpart of the extended interface problems DD, NN, DN and ND.

It is well known (see, e.g., [18, pag. 62], [22]) that $S_{2, \delta}^{0}$ is an optimal preconditioner of $S_{\delta}^{0}$, in the sense that there exists a positive constant $C_{0}$ independent of $\delta$, but depending on problem data (e.g., $\Omega, \nu, \mathbf{b}, b_{0}$ ) such that

$$
\mathcal{K}\left(\left(S_{2, \delta}^{0}\right)^{-1} S_{\delta}^{0}\right) \leqslant C_{0}
$$

where $\mathcal{K}(A)=\left(\max _{i}\left|\lambda_{i}(A)\right|\right) /\left(\min _{i}\left|\lambda_{i}(A)\right|\right)$ is the iterative condition number of a generic square real matrix $A$ whose eigenvalues are $\lambda_{i}(A) \in \mathbb{C}$.

Following [18] it is possible to prove that $S_{2, \delta}^{0}$ is an optimal preconditioner of $S_{\delta}$ with respect to the discretization, i.e., there exists $C_{1}>0$ independent of $\delta$, but depending on problem data (e.g., $\left.\Omega, \nu, \mathbf{b}, b_{0}\right)$ such that

$$
\mathcal{K}\left(\left(S_{2, \delta}^{0}\right)^{-1} S_{\delta}\right) \leqslant C_{1}
$$


Numerical results that confirm (5.11) and (5.12) are shown in Tables 5.1, 5.3, 5.5 (columns SP0 and SP, respectively), where few Preconditioned Bi-CGStab (PBi-CGStab) iterations are sufficient to converge to the required tolerance for all used discretizations. Experimental results show that in general $C_{1} \geqslant C_{0}$.

Let us now consider the extended interface Dirichlet-Dirichlet problem (5.4)-(5.8). We take a $2 \times 2$-block decomposition of $A_{D D, \delta}^{\alpha}$, as drawn by the continuous lines in (5.8). The construction of an optimal preconditioner for problem (5.4) is based on the construction of an optimal preconditioner $P_{\Sigma_{D D, \delta}}$ for the Schur complement $\Sigma_{D D, \delta}^{\alpha}$ of the matrix $A_{D D, \delta}^{\alpha}$, with respect to the $(2,2)$-block $S_{2, \delta}^{\alpha}$.

Such idea has been proposed in [22, Sect. 2.3.1] in the context of domain decomposition methods for elliptic problems.

Since $M_{\delta}^{\text {in }}$ is non-singular, it is easy to see that

$$
\Sigma_{D D, \delta}^{\alpha}=S_{2, \delta}^{\alpha}+\left[\begin{array}{cc}
\alpha S_{1, \delta}^{\text {in }} & 0 \\
S_{1, \delta}^{\text {out }} & 0
\end{array}\right]=\alpha S_{\delta}+(1-\alpha) S_{\delta}^{0}
$$

where $S_{\delta}$ and $S_{\delta}^{0}$ are the matrices introduced in (5.2) and (5.3), respectively. Thus, we set $P_{\Sigma_{D D, \delta}}=S_{2, \delta}^{0}$ and, owing to both (5.11) and (5.12), $P_{\Sigma_{D D, \delta}}$ is an optimal preconditioner for $\Sigma_{D D, \delta}^{\alpha}$, i.e.,

$$
\mathcal{K}\left(P_{\Sigma_{D D, \delta}}^{-1} \Sigma_{D D, \delta}^{\alpha}\right) \leqslant C_{\alpha}, \quad \text { for } \alpha=0,1
$$

Now, we proceed as follows. Since $A_{D D, \delta}^{\alpha}=L_{\delta}^{\alpha} U_{D D, \delta}^{\alpha}$, with

$$
L_{\delta}^{\alpha}=\left[\begin{array}{cc|cc}
I & 0 & 0 & 0 \\
0 & I & 0 & 0 \\
\hline-I & \alpha S_{1, \delta}^{\text {in }} & I & 0 \\
0 & S_{1, \delta}^{\text {out }} & 0 & I
\end{array}\right], \quad U_{D D, \delta}^{\alpha}=\left[\begin{array}{cc|cc}
\alpha S_{1, \delta}^{\text {in }} & M_{\delta}^{\text {in }} & 0 & 0 \\
I & 0 & -I & 0 \\
\hline 0 & 0 & \Sigma_{D D, \delta}^{\alpha}
\end{array}\right] \text {, }
$$

we define the left preconditioner $P_{D D, \delta}^{\alpha}$ for $A_{D D, \delta}^{\alpha}$ as

$$
P_{D D, \delta}^{\alpha}=L_{\delta}^{\alpha} \widetilde{U}_{D D, \delta}^{\alpha}, \quad \text { with } \quad \widetilde{U}_{D D, \delta}^{\alpha}=\left[\begin{array}{cc|cc}
\alpha S_{1, \delta}^{i n} & M_{\delta}^{i n} & 0 & 0 \\
I & 0 & -I & 0 \\
\hline 0 & 0 & P_{\Sigma_{D D, \delta}}
\end{array}\right]
$$

In fact, $\tilde{U}_{D D}^{\alpha}$ differs from $U_{D D}^{\alpha}$ only in the (2,2)-block, where the Schur complement matrix $\Sigma_{D D, \delta}^{\alpha}$ has been replaced by its preconditioner $P_{\Sigma_{D D, \delta}}$.

The following theorem holds:

Theorem 5.1. Let $A \in \mathbb{R}^{n \times n}$ be non-singular, $L, U \in \mathbb{R}^{n \times n}$ be $(2 \times 2)$-block triangular matrices lower and upper, respectively, such that $A=L U$ and

$$
U=\left[\begin{array}{ll}
U_{11} & U_{12} \\
0 & U_{22}
\end{array}\right],
$$

where $U_{11}, U_{22}$ are square blocks. If there exists a square non-singular matrix $P_{22}$ of the same dimension of $U_{22}$ and a positive constant $C$ such that

$$
\mathcal{K}\left(P_{22}^{-1} U_{22}\right) \leqslant C
$$


then the matrix $P=L \widetilde{U}$, with

$$
\widetilde{U}=\left[\begin{array}{ll}
U_{11} & U_{12} \\
0 & P_{22}
\end{array}\right]
$$

satisfies the bound

$$
\mathcal{K}\left(P^{-1} U\right) \leqslant C
$$

Proof. Since $A$ is non-singular, both $U$ and $U_{k k}$ (for $k=1,2$ ) are non-singular and

$$
\widetilde{U}^{-1}=\left[\begin{array}{ll}
U_{11}^{-1} & -U_{11}^{-1} U_{12} P_{22}^{-1} \\
0 & P_{22}^{-1}
\end{array}\right], \quad P^{-1} A=\widetilde{U}^{-1} U=\left[\begin{array}{ll}
I & U_{11}^{-1} U_{12}\left(I-P_{22}^{-1} U_{22}\right) \\
0 & P_{22}^{-1} U_{22}
\end{array}\right],
$$

that is, the spectrum of $P^{-1} A$ coincides with that of $P_{22}^{-1} U_{22}$ plus the eigenvalue $\lambda=1$.

Indeed $\lambda=1$ is an eigenvalue of $P_{22}^{-1} U_{22}$, too. As a matter of fact, the eigenvector $\mathbf{v}$ of $P^{-1} A$ associated to $\lambda=1$ satisfies the matrix equation

$$
A \mathbf{v}=P \mathbf{v} \Leftrightarrow U \mathbf{v}=\widetilde{U} \mathbf{v}
$$

if and only if

$$
\left[\begin{array}{ll}
U_{11} & U_{12} \\
0 & U_{22}
\end{array}\right]\left[\begin{array}{l}
\mathbf{v}_{1} \\
\mathbf{v}_{2}
\end{array}\right]=\left[\begin{array}{ll}
U_{11} & U_{12} \\
0 & P_{22}
\end{array}\right]\left[\begin{array}{l}
\mathbf{v}_{1} \\
\mathbf{v}_{2}
\end{array}\right] \Leftrightarrow U_{22} \mathbf{v}_{2}=P_{22} \mathbf{v}_{2}
$$

that is $\lambda=1$ is an eigenvalue of $P_{22}^{-1} U_{22}$, provided that $\mathbf{v}_{2}$ is not null. It follows that $\lambda_{i}\left(P^{-1} A\right)=\lambda_{i}\left(P_{22}^{-1} U_{22}\right)$ and

$$
\mathcal{K}\left(P^{-1} A\right)=\mathcal{K}\left(P_{22}^{-1} U_{22}\right) .
$$

This concludes the proof.

The next result follows from Theorem 5.1 and states that the matrix $P_{D D, \delta}^{\alpha}$ defined in (5.16) is an optimal preconditioner for $A_{D D, \delta}^{\alpha}$ with respect to the discretization.

Theorem 5.2. We have

$$
\mathcal{K}\left(\left(P_{D D, \delta}^{\alpha}\right)^{-1} A_{D D, \delta}^{\alpha}\right) \leqslant C_{\alpha}
$$

where $C_{\alpha}$ are the positive constants independent of $\delta$, introduced in (5.11) and (5.12).

By considering now the finite dimensional counterpart of the other three formulations, Neumann-Neumann (4.19), Dirichlet-Neumann (4.20) and Neumann-Dirichlet (4.21), and still extracting the Schur complement matrix, again with respect to the $(2,2)$-block, we obtain preconditioners also for the corresponding matrices $A_{N N, \delta}^{\alpha}, A_{D N, \delta}^{\alpha}$ and $A_{N D, \delta}^{\alpha}$. More precisely, the Schur complements of $A_{N N, \delta}^{\alpha}, A_{D N, \delta}^{\alpha}, A_{N D, \delta}^{\alpha}$ and their optimal preconditioners are

$$
\begin{array}{lll}
\Sigma_{N N, \delta}^{\alpha}=\Sigma_{D D, \delta}^{\alpha}\left(S_{2, \delta}^{\alpha}\right)^{-1} M_{\delta}, & \Sigma_{D N, \delta}^{\alpha}=\Sigma_{N N, \delta}^{\alpha}, & \Sigma_{N D, \delta}^{\alpha}=\Sigma_{D D, \delta}^{\alpha} \\
P_{\Sigma_{N N, \delta}}^{\alpha}=S_{2, \delta}^{0}\left(S_{2, \delta}^{\alpha}\right)^{-1} M_{\delta}, & P_{\Sigma_{D N, \delta}}^{\alpha}=P_{\Sigma_{N N, \delta}}^{\alpha}, & P_{\Sigma_{N D, \delta}}=P_{\Sigma_{D D, \delta}} .
\end{array}
$$

Remark 5.3. In view of Remark 4.1 it is noteworthy noticing that $S_{2, \delta}$ is not always invertible. From now on, when we deal with $S_{2, \delta}^{-1}$ we suppose that the vector field b satisfies the smallness assumption (2.24). 
We write

$$
\begin{aligned}
& A_{N N, \delta}^{\alpha}=L_{\delta}^{\alpha} U_{N N, \delta}^{\alpha}, \quad A_{D N, \delta}^{\alpha}=L_{\delta}^{\alpha} U_{D N, \delta}^{\alpha}, \quad A_{N D, \delta}^{\alpha}=L_{\delta}^{\alpha} U_{N D, \delta}^{\alpha} \\
& P_{N N, \delta}^{\alpha}=L_{\delta}^{\alpha} \widetilde{U}_{N N, \delta}^{\alpha}, \quad P_{D N, \delta}^{\alpha}=L_{\delta}^{\alpha} \widetilde{U}_{D N, \delta}^{\alpha}, \quad P_{N D, \delta}^{\alpha}=L_{\delta}^{\alpha} \widetilde{U}_{N D, \delta}^{\alpha}
\end{aligned}
$$

where, in all cases, $L_{\delta}^{\alpha}$ is the matrix defined in (5.15), while

$$
\begin{aligned}
& U_{N N, \delta}^{\alpha}=\left[\begin{array}{cc|cc}
\alpha M_{\delta}^{i n} & M_{\delta}^{i n} & 0 & 0 \\
\left(S_{1, \delta}^{i n}\right)^{-1} M_{\delta}^{i n} & 0 & -R_{2}^{i n}\left(S_{2, \delta}^{\alpha}\right)^{-1} M_{\delta} \\
\hline 0 & 0 & \Sigma_{N N, \delta}^{\alpha}
\end{array}\right] \\
& \widetilde{U}_{N N, \delta}^{\alpha}=\left[\begin{array}{cc|lc}
\alpha M_{\delta}^{i n} & M_{\delta}^{i n} & 0 & 0 \\
\left(S_{1, \delta}^{i n}\right)^{-1} M_{\delta}^{i n} & 0 & -R_{2}^{i n}\left(S_{2, \delta}^{\alpha}\right)^{-1} M_{\delta} \\
\hline 0 & 0 & P_{\Sigma_{N N, \delta}}^{\alpha}
\end{array}\right],
\end{aligned}
$$

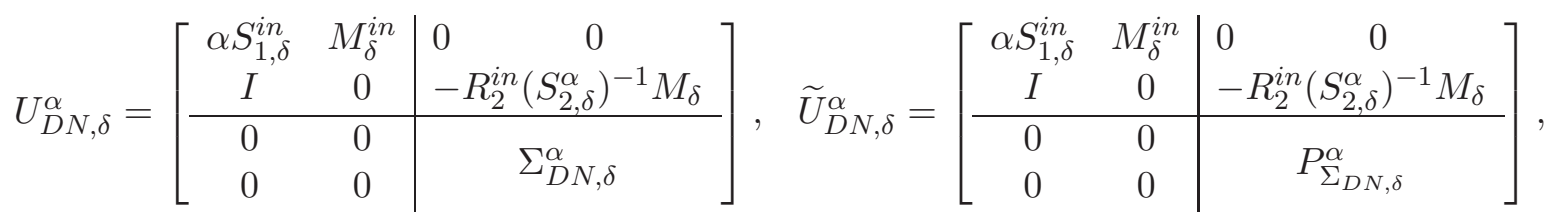

$$
\begin{aligned}
& U_{N D, \delta}^{\alpha}=\left[\begin{array}{cc|cc}
\alpha M_{\delta}^{i n} & M_{\delta}^{i n} & 0 & 0 \\
\left(S_{1, \delta}^{i n}\right)^{-1} M_{\delta}^{i n} & 0 & -I & 0 \\
\hline 0 & 0 & \Sigma_{N D, \delta}^{\alpha}
\end{array}\right], \quad \widetilde{U}_{N D, \delta}^{\alpha}=\left[\begin{array}{cc|cc}
\alpha M_{\delta}^{i n} & M_{\delta}^{i n} & 0 & 0 \\
\left(S_{1, \delta}^{i n}\right)^{-1} M_{\delta}^{i n} & 0 & -I & 0 \\
\hline 0 & 0 & 0 & P_{\Sigma_{N D, \delta}}
\end{array}\right] .
\end{aligned}
$$

The same conclusion of Theorem 5.2 can be stated also for NN, DN and ND formulations, more precisely.

Theorem 5.3. We have

$$
\mathcal{K}\left(\left(P_{N D, \delta}^{\alpha}\right)^{-1} A_{N D, \delta}^{\alpha}\right) \leqslant C_{\alpha},
$$

where $C_{\alpha}$ is the constant introduced in (5.12). Moreover, if $S_{2, \delta}$ is invertible (for that it suffices that (2.24) be satisfied) when $\alpha=1$, then

$$
\mathcal{K}\left(\left(P_{N N, \delta}^{\alpha}\right)^{-1} A_{N N, \delta}^{\alpha}\right) \leqslant C_{\alpha}, \quad \mathcal{K}\left(\left(P_{D N, \delta}^{\alpha}\right)^{-1} A_{D N, \delta}^{\alpha}\right) \leqslant C_{\alpha} .
$$

Proof. Estimate (5.23) immediately follows from the equality $\Sigma_{N D, \delta}^{\alpha}=\Sigma_{D D, \delta}^{\alpha}$. By (5.21), it holds

$$
\left(P_{\Sigma_{N N, \delta}}^{\alpha}\right)^{-1} \Sigma_{N N, \delta}^{\alpha}=M_{\delta}^{-1} S_{2, \delta}^{\alpha}\left(S_{2, \delta}^{0}\right)^{-1} \Sigma_{D D, \delta}^{\alpha}\left(S_{2, \delta}^{\alpha}\right)^{-1} M_{\delta}
$$

that is, if $S_{2, \delta}^{\alpha}$ is invertible, then $\left(P_{\Sigma_{N N, \delta}}^{\alpha}\right)^{-1} \Sigma_{N N, \delta}^{\alpha}$ is similar to $P_{\Sigma_{D D, \delta}}^{-1} \sum_{D D, \delta}^{\alpha}$. The same conclusion holds for $\Sigma_{D N, \delta}^{\alpha}$.

Remark 5.4. We notice that, even if $\left(S_{2, \delta}^{\alpha}\right)^{-1}$ shows up in all Schur complement matrices defined in (5.21) and in both $\tilde{U}_{N N, \delta}^{\alpha}$ and $\tilde{U}_{D N, \delta}^{\alpha}$, actually only the inversion of $S_{2, \delta}^{0}$ is required when solving the linear systems $P \mathbf{z}=\mathbf{r}$. This means that during the preconditioner step we do not solve elliptic problems with interface condition $\nu \partial u_{2} / \partial \mathbf{n}_{2}-\mathbf{b} \cdot \mathbf{n}_{2} u_{2}=\phi$ on $\Gamma^{i n}$, that might be ill-posed when $\alpha=1$ and (2.24) does not hold. 


\begin{tabular}{r|c|c|c|c|c}
\hline & & \multicolumn{4}{|c}{$\alpha=0$} \\
\hline$N$ & SP0 & DD & NN & DN & ND \\
\hline 8 & 1 & 2 & 3 & 3 & 2 \\
16 & 1 & 2 & 3 & 3 & 2 \\
24 & 1 & 2 & 3 & 3 & 2 \\
28 & 1 & 2 & 3 & 3 & 2 \\
\hline \hline & & \multicolumn{5}{|c}{$\alpha=1$} \\
\hline$N$ & SP & DD & NN & DN & ND \\
\hline 8 & 2 & 2 & 2 & 2 & 2 \\
16 & 1 & 2 & 2 & 2 & 2 \\
24 & 1 & 2 & 2 & 2 & 2 \\
28 & 1 & 2 & 2 & 2 & 2 \\
\hline
\end{tabular}

\begin{tabular}{r|c|c|c|c|c}
\hline & & \multicolumn{4}{|c}{$\alpha=0$} \\
\hline$M_{x}=M_{y}$ & SP0 & DD & NN & DN & ND \\
\hline 8 & 1 & 2 & 3 & 3 & 2 \\
16 & 1 & 2 & 3 & 3 & 2 \\
20 & 1 & 2 & 3 & 3 & 2 \\
24 & 1 & 2 & 3 & 3 & 2 \\
\hline \hline & & \multicolumn{5}{|c}{$\alpha=1$} \\
\hline$M_{x}=M_{y}$ & SP & DD & NN & DN & ND \\
\hline 8 & 1 & 2 & 2 & 2 & 2 \\
16 & 1 & 2 & 2 & 2 & 2 \\
20 & 1 & 2 & 2 & 2 & 2 \\
24 & 1 & 2 & 2 & 2 & 2 \\
\hline
\end{tabular}

Table 5.1. Test case \#1. PBi-CGStab iterations needed to solve the preconditioned finite dimensional systems DD (5.4), NN (5.5), DN (5.6), ND (5.7), and the Steklov-Poincaré equations SP0 (5.3) and SP (5.2). The viscosity is $\nu=0.01$. The discretizations coincide with those used to provide the results of Fig. 5.2. At right, the total number of spectral elements in each subdomain $\Omega_{k}$ is $M=M_{x} \times M_{y}$

\begin{tabular}{c|c|c|c|c|c}
\hline & & \multicolumn{4}{|c}{$\alpha=0$} \\
\hline$\nu$ & SP0 & DD & NN & DN & ND \\
\hline $10^{-1}$ & 1 & 2 & 3 & 3 & 2 \\
$10^{-2}$ & 1 & 2 & 3 & 3 & 2 \\
$10^{-3}$ & 1 & 2 & 3 & 3 & 2 \\
$10^{-4}$ & 1 & 2 & 3 & 3 & 2 \\
\hline
\end{tabular}

\begin{tabular}{c|c|c|c|c|c}
\hline & & \multicolumn{4}{|c}{$\alpha=1$} \\
\hline$\nu$ & SP & DD & NN & DN & ND \\
\hline $10^{-1}$ & 1 & 2 & 2 & 2 & 2 \\
$10^{-2}$ & 1 & 2 & 2 & 2 & 2 \\
$10^{-3}$ & 1 & 2 & 2 & 2 & 2 \\
$10^{-4}$ & 1 & 2 & 2 & 2 & 2 \\
\hline
\end{tabular}

Table 5.2. Test case \#1. PBi-CGStab iterations needed to solve the preconditioned finite dimensional systems DD (5.4), NN (5.5), DN (5.6), ND (5.7), and the Steklov Poincaré equations SP0 (5.3) and SP (5.2)

Numerical results shown in Tables 5.1-5.6 are obtained by using preconditioners defined in (5.16) and (5.22). We note that for Test case \#1, the preconditioned version of both NN and DN does not suffer from instability for all considered viscosity, in spite of the corresponding unpreconditioned version when $\alpha=1$ (see Fig. 5.3 and Tab. 5.2).

In all the test cases the number of Preconditioned Bi-CGStab (PBi-CGStab) iterations is bounded from above for both Steklov-Poincaré and extended forms, independently of the used discretization (see Tables 5.1, 5.3, 5.5). The discrete Steklov-Poincaré form SP0 (5.3) provides the lower number of PBi-CGStab iterations.

In Tables 5.2, 5.4 and 5.6, the number of PBi-CGStab iterations is shown for vanishing viscosity. We notice that it is bounded from above in all cases with the exception of the Test case \#3, approaches $\mathrm{NN}$ and $\mathrm{DN}$ when $\alpha=1$, and $\nu=10^{-4}$. Here the same instabilities shown in Fig. 5.4 (left) occur and we conjecture that, for this particular choice of the vector field $\mathbf{b}$, the non-coercivity of the Steklov-Poincaré $\mathcal{S}_{2}$ is responsible of it even in the case of preconditioned systems.

We conclude that in general, the set of interface conditions IC2 (2.11) provides the best efficient approaches in terms of PBi-CGStab iterations and they do not suffer from instabilities in the case of advection-dominated problems.

5.3.1. Computational cost per iteration The results of Tables 5.1, 5.3, 5.5 confirm optimality properties for all the preconditioners presented in Sec. 5.3. Therefore, to sort out the "best" method, a remark on the computational impact per iteration is necessary. 


\begin{tabular}{r|c|c|c|c|c}
\hline & & \multicolumn{4}{|c}{$\alpha=0$} \\
\hline$N$ & SP0 & DD & NN & DN & ND \\
\hline 8 & 3 & 5 & 4 & 4 & 5 \\
12 & 3 & 5 & 4 & 4 & 5 \\
16 & 3 & 5 & 4 & 4 & 5 \\
20 & 3 & 5 & 3 & 3 & 5 \\
\hline \hline & & \multicolumn{5}{|c}{$\alpha=1$} \\
\hline$N$ & SP & DD & NN & DN & ND \\
\hline 8 & 4 & 5 & 6 & 6 & 5 \\
12 & 5 & 6 & 6 & 6 & 6 \\
16 & 5 & 6 & 6 & 6 & 6 \\
20 & 5 & 6 & 6 & 6 & 6 \\
\hline
\end{tabular}

\begin{tabular}{r|c|c|c|c|c}
\hline & & \multicolumn{4}{|c}{$\alpha=0$} \\
\hline$M_{x}=M_{y}$ & SP0 & DD & NN & DN & ND \\
\hline 4 & 3 & 5 & 4 & 4 & 5 \\
8 & 3 & 5 & 4 & 4 & 5 \\
12 & 3 & 5 & 4 & 4 & 5 \\
16 & 3 & 5 & 4 & 4 & 5 \\
\hline \hline & & \multicolumn{5}{|c}{$\alpha=1$} \\
\hline$M_{x}=M_{y}$ & SP & DD & NN & DN & ND \\
\hline 4 & 4 & 6 & 6 & 6 & 6 \\
8 & 4 & 7 & 7 & 7 & 6 \\
12 & 5 & 6 & 7 & 8 & 6 \\
16 & 5 & 6 & 8 & 8 & 6 \\
\hline
\end{tabular}

Table 5.3. Test case \#2. PBi-CGStab iterations needed to solve the preconditioned finite dimensional systems DD (5.4), NN (5.5), DN (5.6), ND (5.7), and the Steklov-Poincaré equations SP0 (5.3) and SP (5.2). The viscosity is $\nu=0.01$. At left, $M_{x}=M_{y}=4$ in both $\Omega_{1}$ and $\Omega_{2}$. At right, $N=6$ in each element, the total number of spectral elements in each subdomain $\Omega_{k}$ is $M=M_{x} \times M_{y}$

\begin{tabular}{c|c|c|c|c|c}
\hline & & \multicolumn{4}{|c}{$\alpha=0$} \\
\hline$\nu$ & SP0 & DD & NN & DN & ND \\
\hline $10^{-1}$ & 3 & 5 & 3 & 3 & 5 \\
$10^{-2}$ & 3 & 5 & 4 & 4 & 5 \\
$10^{-3}$ & 3 & 5 & 3 & 3 & 4 \\
$10^{-4}$ & 5 & 6 & 5 & 5 & 6 \\
\hline
\end{tabular}

\begin{tabular}{c|c|c|c|c|c}
\hline & & \multicolumn{4}{|c}{$\alpha=1$} \\
\hline$\nu$ & SP & DD & NN & DN & ND \\
\hline $10^{-1}$ & 5 & 5 & 6 & 6 & 5 \\
$10^{-2}$ & 5 & 6 & 6 & 6 & 6 \\
$10^{-3}$ & 6 & 6 & 7 & 7 & 6 \\
$10^{-4}$ & 7 & 7 & 9 & 8 & 7 \\
\hline
\end{tabular}

Table 5.4. Test case \#2. PBi-CGStab iterations needed to solve the preconditioned finite dimensional systems DD (5.4), NN (5.5), DN (5.6), ND (5.7), and the Steklov Poincaré equations SP0 (5.3) and SP (5.2)

\begin{tabular}{r|c|c|c|c|c}
\hline & & \multicolumn{4}{|c}{$\alpha=0$} \\
\hline$N$ & SP0 & DD & NN & DN & ND \\
\hline 8 & 3 & 4 & 4 & 4 & 4 \\
12 & 3 & 5 & 4 & 4 & 5 \\
16 & 3 & 4 & 3 & 3 & 4 \\
20 & 3 & 4 & 3 & 3 & 4 \\
\hline \hline & & \multicolumn{5}{|c}{$\alpha=1$} \\
\hline$N$ & SP & DD & NN & DN & ND \\
\hline 8 & 6 & 6 & 7 & 7 & 8 \\
12 & 6 & 6 & 7 & 7 & 7 \\
16 & 6 & 6 & 6 & 6 & 6 \\
20 & 5 & 6 & 6 & 6 & 6 \\
\hline
\end{tabular}

\begin{tabular}{r|c|c|c|c|c}
\hline & & \multicolumn{4}{|c}{$\alpha=0$} \\
\hline$M_{x}=M_{y}$ & SP0 & DD & NN & DN & ND \\
\hline 4 & 3 & 5 & 4 & 4 & 5 \\
8 & 3 & 5 & 4 & 4 & 5 \\
12 & 3 & 5 & 4 & 5 & 5 \\
16 & 3 & 4 & 4 & 4 & 4 \\
\hline \hline & & \multicolumn{5}{|c}{$\alpha=1$} \\
\hline$M_{x}=M_{y}$ & SP & DD & NN & DN & ND \\
\hline 4 & 6 & 7 & 7 & 7 & 6 \\
8 & 7 & 7 & 8 & 8 & 7 \\
12 & 7 & 8 & 9 & 9 & 7 \\
16 & 6 & 7 & 8 & 8 & 7 \\
\hline
\end{tabular}

Table 5.5. Test case \#3. PBi-CGStab iterations needed to solve the preconditioned finite dimensional systems DD (5.4), NN (5.5), DN (5.6), ND (5.7), and the Steklov-Poincaré equations SP0 (5.3) and SP (5.2). The viscosity is $\nu=0.01$. At left, $M_{x}=M_{y}=4$ in both $\Omega_{1}$ and $\Omega_{2}$. At right, $N=6$ in each element, the total number of spectral elements in each subdomain $\Omega_{k}$ is $M=M_{x} \times M_{y}$ 


\begin{tabular}{r|c|c|c|c|c}
\hline & & \multicolumn{4}{|c}{$\alpha=0$} \\
\hline$\nu$ & SP0 & DD & NN & DN & ND \\
\hline $10^{-1}$ & 2 & 3 & 3 & 3 & 3 \\
$10^{-2}$ & 3 & 4 & 3 & 3 & 4 \\
$10^{-3}$ & 3 & 5 & 4 & 4 & 5 \\
$10^{-4}$ & 5 & 6 & 5 & 5 & 6 \\
\hline
\end{tabular}

\begin{tabular}{r|c|c|c|c|c}
\hline & & \multicolumn{4}{|c}{$\alpha=1$} \\
\hline$\nu$ & SP & DD & NN & DN & ND \\
\hline $10^{-1}$ & 4 & 5 & 5 & 5 & 5 \\
$10^{-2}$ & 6 & 6 & 6 & 6 & 6 \\
$10^{-3}$ & 7 & 9 & 8 & 9 & 8 \\
$10^{-4}$ & 11 & 8 & $>40$ & $>40$ & 9 \\
\hline
\end{tabular}

Table 5.6. Test case \#3. PBi-CGStab iterations needed to solve the preconditioned finite dimensional systems DD (5.4), NN (5.5), DN (5.6), ND (5.7), and the Steklov Poincaré equations SP0 (5.3) and SP (5.2). When $\nu \lesssim 10^{-4}$ and $\alpha=1$ both NN and DN forms suffer from the ill-posedness of Steklov-Poincaré operator $\mathcal{S}_{2}$

Each PBi-CGStab iteration requires to compute 2 matrix-vector products and to solve 2 linear systems on the preconditioner, then we analyze these operations.

First of all we recall that, thanks to the use of Gauss-Lobatto numerical integration, the spectral-element mass matrices are diagonal, so that either multiplication or inversion of mass matrices is a low-cost operation. Recalling that the local Steklov-Poincaré operators $\mathcal{S}_{k}^{\alpha}$ (for $k=1,2$ ) realize Dirichlet to Neumann maps, it follows that the action of $S_{1, \delta}^{\alpha}$ requires the solution of a hyperbolic problem in $\Omega_{1}$, while the action of $S_{2, \delta}^{\alpha}$ requires the solution of an elliptic problem in $\Omega_{2}$. Both the inverse operators $\left(S_{1, \delta}^{\alpha}\right)^{-1}$ and $\left(S_{2, \delta}^{\alpha}\right)^{-1}$ realize Neumann to Dirichlet maps and they require the same computational cost of the corresponding operators $S_{1, \delta}^{\alpha}$ and $S_{2, \delta}^{\alpha}$, respectively. The approximation of local hyperbolic and elliptic problems, is the more expensive step of the process, so we measure the computational complexity of either one Matrix-Vector Product (MVP) and one Linear System whose matrix is the Preconditioner (LSP) in terms of number of hyperbolic and elliptic problems to be solved in $\Omega_{1}$ and $\Omega_{2}$, respectively.

In Table 5.7 we summarize the number of hyperbolic and elliptic problems to be solved for implementing one MVP and one LSP and one PBi-CGStab iteration.

\begin{tabular}{l|l|l|l}
\hline & 1 MVP & 1 LSP & 1 PBi-CGStab it \\
\hline \hline SP0 & 1 ell +1 hyp & 1 ell & 4 ell +2 hyp \\
SP & 1 ell +1 hyp & 1 ell & 4 ell +2 hyp \\
\hline DD $(\alpha=0)$ & 1 ell +1 hyp & 1 ell +1 hyp & 4 ell +4 hyp \\
DN $(\alpha=0)$ & 1 ell +1 hyp & 1 ell +1 hyp & 4 ell +4 hyp \\
ND $(\alpha=0)$ & 1 ell +1 hyp & 1 ell +2 hyp & 4 ell +6 hyp \\
NN $(\alpha=0)$ & 1 ell +1 hyp & 1 ell +2 hyp & 4 ell +6 hyp \\
\hline DD $(\alpha=1)$ & 1 ell +1 hyp & 1 ell +2 hyp & 4 ell +6 hyp \\
ND $(\alpha=1)$ & 1 ell +1 hyp & 1 ell +2 hyp & 4 ell +6 hyp \\
DN $(\alpha=1)$ & 1 ell +1 hyp & 2 ell +2 hyp & 6 ell +6 hyp \\
NN $(\alpha=1)$ & 1 ell +1 hyp & 2 ell +2 hyp & 6 ell +6 hyp \\
\hline
\end{tabular}

Table 5.7. Computational cost of each approach in terms of the number of elliptic (ell) and hyperbolic (hyp) subproblems

In conclusion, Steklov-Poincaré approaches provide the "best" performing PBi-CGStab iteration, DD and ND forms with $\alpha=0$ follow, while DN and NN when $\alpha=1$ are the most expensive ones. 

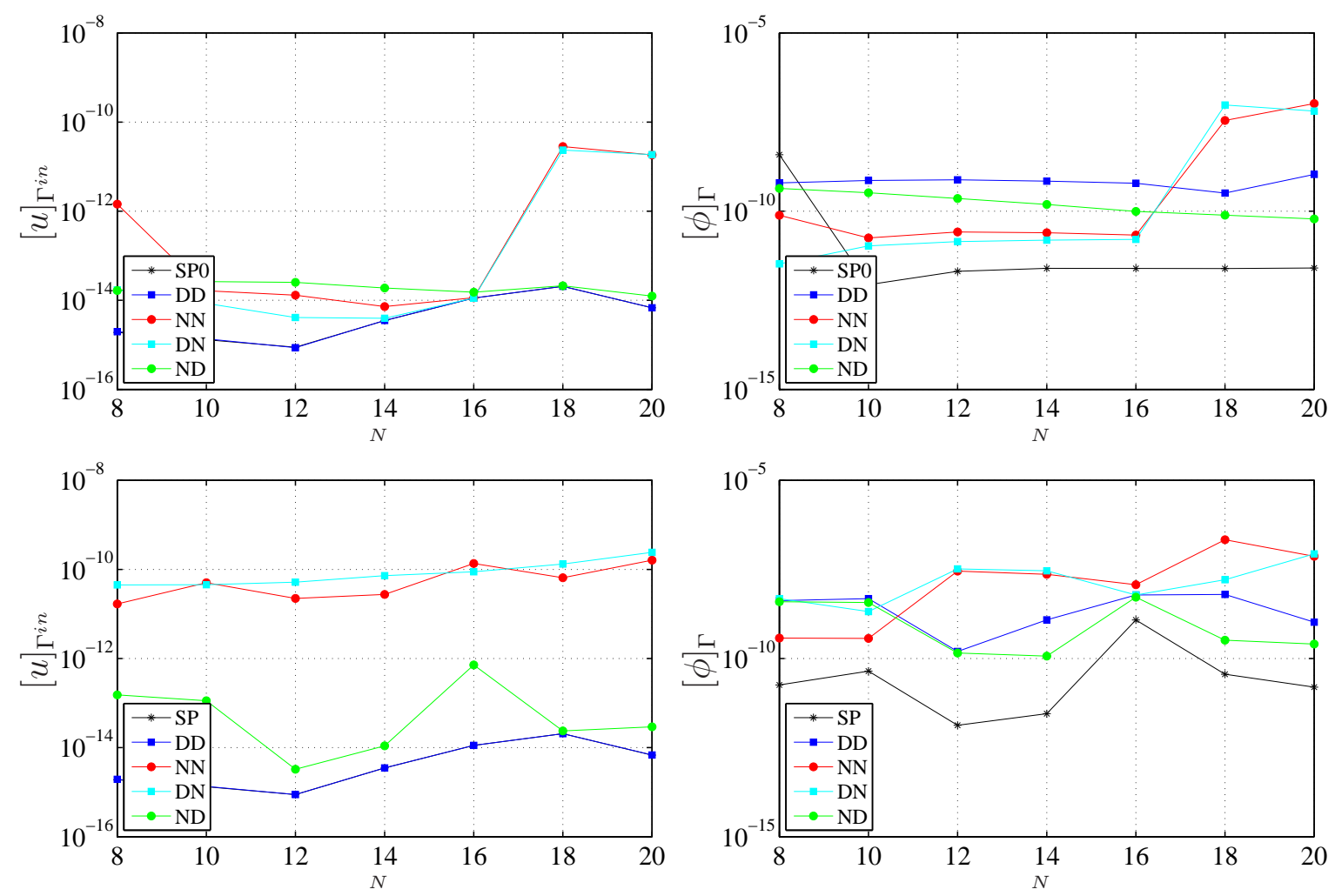

Figure 5.5. Test case \#2. Conforming coupling. At left $[u]_{\Gamma^{i n}}$, at right $[\phi]_{\Gamma}$ versus the polynomial degree $N$. The viscosity is $\nu=0.01$. The discretization is that used to provide the results of Tab. 5.3 left. At top, $\alpha=0$, at bottom, $\alpha=1$

5.3.2. Accuracy comparison. We compare now all formulations for what concerns the jumps of both solutions and fluxes across the interface.

Recalling that no continuity constraint is imposed on $\Gamma^{\text {out }}$, we measure the jump between $u_{1}$ and $u_{2}$ on $\Gamma^{i n}$, while the jump of fluxes is measured on the whole interface $\Gamma$. Then we set

$$
[u]_{\Gamma^{i n}}=\left\|u_{1, \delta}-u_{2, \delta}\right\|_{\delta, L^{2}\left(\Gamma^{i n}\right)} \quad[\phi]_{\Gamma}=\left\|\mathbf{b} \cdot \mathbf{n}_{\Gamma} u_{1, \delta}+\nu \frac{\partial u_{2, \delta}}{\partial \mathbf{n}_{\Gamma}}-\mathbf{b} \cdot \mathbf{n}_{\Gamma} u_{2, \delta}\right\|_{\delta, L^{2}(\Gamma)}
$$

where the sub-index $\delta$ in $\|\cdot\|_{\delta \text {, }}$ means that we have computed discrete norms on the nodes of either $\mathcal{T}_{2} \cap \Gamma^{\text {in }}$ or $\mathcal{T}_{2} \cap \Gamma$.

For what concerns the trace jump, we observe that both Steklov-Poincaré SP and SP0, and extended DD approaches provide the smaller jumps and perform similarly. On the contrary, extended DN and NN approaches produce the largest trace jump (see Fig. 5.5, left). For what concerns flux jumps, the Steklov-Poincaré approach SP0 is the most accurate one, followed by SP and by the other XIP forms, without a clear ranking. On the whole we can say that both NN and DN provide the largest flux jumps when $\alpha=1$ (see Fig. 5.5, right).

Similar conclusions can be drawn when addressing the other analyzed test cases. 


\section{Conclusions}

One of the contributions of the present work has been to formulate an extended interface problem (XIP) to treat the domain decomposition problem when coupling heterogeneous models governed by partial differential equations.In order to do that it was necessary to recast the problem as a saddle point formulation. Specifically, this has been carried out in such a way that it was possible to switch between two different formulations by choosing a parameter $\alpha \in\{0,1\}$ (see (3.4)). These two cases yield different Euler-Lagrange equations, which in turn have an impact in the performance of the numerical methods for solving the problem in an iterative manner.

Furthermore, the analysis of these formulations allowed us to provide a guidance with respect to the choice of the XIP form such that the performance of the iterative methods and the solution obtained after convergence of the iterative methods are better. As well, another novelty of the work that must be highlighted is the development of preconditioners for the different XIP. No preconditioners for extended problems have been developed so far and it has been proved that the proposed preconditioners have good properties. When comparing the classical SP0 with the XIP notice that in the latter it is possible to get, after convergence, the consistent flux of the problem directly from the solution process, since now it is considered an unknown in our problem. Moreover, this extended formulation may be useful when working with different meshes from the underlying subdomains, being this a matter of current research (see [5]).

\section{References}

[1] F. B. Belgacem, The mortar finite element method with Lagrange multipliers, Numer. Math., 84 (1999), no. 2, pp. 173-197.

[2] C. Bernardi, Y. Maday, and A. Patera, A new nonconforming approach to domain decomposition: the mortar element method, in: Nonlinear partial differential equations and their applications. Collège de France Seminar, Vol. XI (Paris, 1989-1991), vol. 299 of Pitman Res. Notes Math. Ser., Longman Sci. Tech., Harlow, 1994, pp. 13-51.

[3] P. Blanco, R. Feijóo, and S. Urquiza, A unified variational approach for coupling 3D-1D models and its blood flow applications, Comput. Methods Appl. Mech. Engrg., 196 (2007), no. 41-44, pp. 4391-4410.

[4] P. Blanco, R. Feijóo, and S. Urquiza, A variational approach for coupling kinematically incompatible structural models, Comput. Methods Appl. Mech. Engrg., 197 (2008), no. 17-18, pp. 1577-1602.

[5] P. Blanco, P. Gervasio, and A. Quarteroni, Extended variational formulation for heterogeneous pde's: nonconforming coupling, Tech. rep., in preparation.

[6] I. Boglaev, The solution of a singularly perturbed convection-diffusion problem by an iterative domain decomposition method, Numer. Algorithms, 31 (2002), no. 1-4, pp. 27-46, numerical methods for ordinary differential equations (Auckland, 2001).

[7] F. Brezzi, On the existence, uniqueness and approximation of saddle-point problems arising from Lagrange multipliers, R.A.I.R.O. Anal. Numér., 8 (R2) (1974), pp. 129-151.

[8] C. Canuto, M. Y. Hussaini, A. Quarteroni, and T. A. Zang, Spectral Methods. Fundamentals in Single Domains, Springer, Heidelberg, 2006.

[9] C. Canuto, M. Y. Hussaini, A. Quarteroni, and T. A. Zang, Spectral Methods. Evolution to Complex Geometries and Applications to Fluid Dynamics, Springer, Heidelberg, 2007.

[10] C. Coclici, G. Moroşanu, and W. Wendland, The coupling of hyperbolic and elliptic boundary value problems with variable coefficients, Math. Methods Appl. Sci., 23 (2000), no. 5, pp. 401-440. 
[11] E. Dubach, Contribution à la résolution des equations fluides en domaine non borné., Ph.D. thesis, Universitè Paris 13, 1993.

[12] R. Durán, Mixed finite element methods, vol. 1939 of Lecture Notes in Mathematics, Springer-Verlag, Berlin, 2008, pp. 1-44, lectures given at the C.I.M.E. Summer School held in Cetraro, June 26-July 1, 2006. Edited by D. Boffi and L. Gastaldi.

[13] A. Ern and J.-L. Guermond, Theory and practice of finite elements, vol. 159 of Applied Mathematical Sciences, Springer-Verlag, New York, 2004.

[14] M. Gander, L. Halpern, and C. Japhet, Optimized Schwarz algorithms for coupling convection and convection-diffusion problems, in: Domain decomposition methods in science and engineering (Lyon, 2000), Theory Eng. Appl. Comput. Methods, Internat. Center Numer. Methods Eng. (CIMNE), Barcelona, 2002, pp. 255-262.

[15] M. Gander, L. Halpern, C. Japhet, and V. Martin, Advection diffusion problems with pure advection approximation in subregions, in: Domain decomposition methods in science and engineering XVI, vol. 55 of Lect. Notes Comput. Sci. Eng., Springer, Berlin, 2007, pp. 239-246.

[16] M. Gander, L. Halpern, C. Japhet, and V. Martin, Viscous problems with inviscid approximations in subregions: a new approach based on operator factorization, in: CANUM 2008, vol. 27 of ESAIM Proc., EDP Sci., Les Ulis, 2009, pp. 272-288.

[17] F. Gastaldi and A. Quarteroni, On the coupling of hyperbolic and parabolic systems: analytical and numerical approach., Appl. Numer. Math., 6 (1989), no. 1, pp. 3-31.

[18] F. Gastaldi, A. Quarteroni, and G. S. Landriani, On the coupling of two dimensional hyperbolic and elliptic equations: analytical and numerical approach, in: Third International Symposium on Domain Decomposition Methods for Partial Differential Equations (J. T.F.Chan, R.Glowinski and O.B.Widlund, eds.), SIAM, Philadelphia, 1990, pp. 22-63.

[19] J.-P. Lohéac, F. Nataf, and M. Schatzman, Parabolic approximations of the convection-diffusion equation, Math. Comp., 60 (1993), no. 202, pp. 515-530.

[20] D. D. Pietro, A. Ern, and J.-L. Guermond, Discontinuous Galerkin methods for anisotropic semidefinite diffusion with advection, SIAM J. Numer. Anal., 46 (2008), no. 2, pp. 805-831.

[21] A. Quarteroni and A. Valli, Numerical Approximation of Partial Differential Equations, Springer Verlag, Heidelberg, 1994.

[22] A. Quarteroni and A. Valli, Domain Decomposition Methods for Partial Differential Equations, Oxford Science Publications, 1999.

[23] W. Rudin, Functional analysis, International Series in Pure and Applied Mathematics, 2nd edn., McGraw-Hill Inc., New York, 1991.

[24] H. van der Vorst, Iterative Krylov methods for large linear systems, vol. 13 of Cambridge Monographs on Applied and Computational Mathematics, Cambridge University Press, Cambridge, 2003.

[25] K. Yosida, Functional Analysis, Springer-Verlag, Berlin, 1974, 4th ed. 